\title{
Counterexamples to Hochschild-Kostant-Rosenberg in characteristic $p$
}

\author{
Benjamin Antieau ${ }^{1}$, Bhargav Bhatt ${ }^{2}$ and Akhil Mathew ${ }^{3}$ \\ ${ }^{1}$ Department of Mathematics, Northwestern University, Evanston, IL 60208; E-mail: antieau @ northwestern.edu. \\ ${ }^{2}$ Department of Mathematics, University of Michigan, Ann Arbor, MI 48109; E-mail: bhattb@umich.edu. \\ ${ }^{3}$ Department of Mathematics, University of Chicago, Chicago, IL 60637; E-mail: amathew @ math.uchicago.edu.
}

Received: 20 October 2019; Accepted: 22 January 2021

2020 Mathematics Subject Classification: 13D03; 14F40; 16E40; 19 D55

\begin{abstract}
We give counterexamples to the degeneration of the Hochschild-Kostant-Rosenberg spectral sequence in characteristic $p$, both in the untwisted and twisted settings. We also prove that the de Rham-HP and crystalline-TP spectral sequences need not degenerate.
\end{abstract}

\section{Introduction}

Let $k$ be a commutative ring and $R$ a commutative flat $k$-algebra. Recall that the Hochschild homology complex $\mathrm{HH}(R / k)$ of $R$ relative to $k$ can be defined as the 'functions on the self-intersection of the diagonal of $\operatorname{Spec}(R) \rightarrow \operatorname{Spec}(k)^{\prime}$; that is, as

$$
\mathrm{HH}(R / k)=R \otimes_{R \otimes_{k} R}^{L} R
$$

The Hochschild-Kostant-Rosenberg (HKR) theorem connects the cohomology groups of this complex to differential forms over $k$ : if $R$ is smooth, there are canonical isomorphisms

$$
H^{-n}(\mathrm{HH}(R / k)) \cong \Omega_{R / k}^{n} .
$$

In characteristic 0 , the formula (1) upgrades to a canonical decomposition (often referred to as the Hodge decomposition) of the Hochschild complex; cf., for instance, [32, Th. 8.6], [20], [43]; this implies that for a smooth variety $X / k$ there are canonical isomorphisms

$$
H^{-n}(\mathrm{HH}(X / k)) \cong \bigoplus_{s-t=n} H^{t}\left(X, \Omega_{X / k}^{s}\right)
$$

In this article, we study to what extent the decomposition (2) might hold when $k$ has positive characteristic $p>0$. More precisely, given a smooth $k$-scheme $X$, the canonicity of the HKR isomorphism (1) already implies that one has an $E_{2}$-spectral sequence, which we call the HKR spectral sequence, of the form

$$
E_{2}^{s, t}=H^{s}\left(X, \Omega_{X / k}^{-t}\right) \Rightarrow H^{s+t} \mathrm{HH}(X / k) .
$$

(C) The Author(s), 2021. Published by Cambridge University Press. This is an Open Access article, distributed under the terms of the Creative Commons Attribution licence (http://creativecommons.org/licenses/by/4.0/), which permits unrestricted re-use, distribution, and reproduction in any medium, provided the original work is properly cited. 
We ask here whether this spectral sequence always has to degenerate at $E_{2}$ (e.g., for smooth proper varieties).

Degeneration of the HKR spectral sequence is known if $X / k$ is smooth proper of dimension $\leqslant p$ by work of Yekutieli [45] (in the case where $\operatorname{dim}(X)<p$, in which case one also gets a canonical decomposition) and by Antieau-Vezzosi [7] (which allows $\operatorname{dim}(X)=p$ as well). Additionally, Rao et al. [34] recently proved that HKR holds for the blowup of a smooth proper $X$ along a smooth closed subscheme $Z$ if and only if it holds for $X$ and $Z$. There are additional examples in which one can verify degeneration; for example, smooth complete intersections in projective space (see [7, Ex. 1.7]). In contrast, we prove the following result.

Theorem 1.1. Let $k$ be a perfect field of characteristic $p>0$. There exists a smooth projective $2 p$ dimensional $k$-scheme $X$ such that the HKR spectral sequence for $X$ does not degenerate, so there can be no Hodge decomposition of $H H(X / k)$. Specifically, we construct such an $X$ for which the differential $d_{p}: H^{0}\left(X, \Omega_{X / k}^{1}\right) \rightarrow H^{p}\left(X, \wedge^{p} L_{X / k}\right)$ in the HKR spectral sequence is nonzero.

We also give related examples where the de Rham-HP and crystalline-TP spectral sequences constructed in [12] are nondegenerate and examples where the crystalline-TP spectral sequence gives a non-split filtration on $H^{*}(\mathrm{TP}(X))$. For details, see Theorem 6.2.

Our method is to understand these spectral sequences in the case where we replace the scheme $X$ by the classifying stack $B G$ of a group scheme $G$. In fact, slightly surprisingly, $G=\mu_{p}$ already leads to Theorem 1.1. In this case, the reason for nondegeneracy of the HKR spectral sequence is relatively easy to describe, at least informally: the Hochschild homology of $B \mu_{p}$ is concentrated in degree 0 (because the category of quasicoherent sheaves on $B \mu_{p}$ or, equivalently, the category of representations of $\mu_{p}$ is just the $p$-fold direct sum of the category of vector spaces), whereas the Hodge cohomology of $B \mu_{p}$ is not concentrated in degree 0 (because there are nontrivial 1-forms on $B \mu_{p}$ arising from the singularities of $\mu_{p}$ as a scheme).

To pass from the stacks discussed above to the examples of Theorem 1.1, we approximate $B G$ by smooth projective varieties; that is, we find maps $X \rightarrow B G$ with $X$ smooth projective such that the pullback map on various cohomology theories considered above is injective. More specifically, we prove the following theorem.

Theorem 1.2. Suppose that $k$ is a perfect field of characteristic $p>0$ and that $G$ is an affine $k$ group scheme that is either finite or geometrically reductive. For any integer $d \geqslant 0$, there exists $a$ smooth projective $k$-scheme $X$ of dimension d together with a map $X \rightarrow B G$ such that the pullback $H^{s}\left(B G, \wedge{ }^{t} L_{B G / k}\right) \rightarrow H^{s}\left(X, \wedge^{t} L_{X / k}\right)$ is injective for $s+t \leqslant d$.

The geometric idea behind finding such approximations goes back to the work of Godeaux and Serre. However, because the relevant group schemes $G$ are not smooth, one runs into possibly singular complete intersections in projective space as intermediate objects in this argument. To handle their cohomology, we prove a version of the weak Lefschetz theorem for Hodge cohomology for such complete intersections.

We also consider the twisted version of this question. Let $X / k$ be a smooth $k$-scheme and let $\alpha \in H^{2}\left(X, \mathbf{G}_{m}\right)$ be a cohomological Brauer class. One constructs a twisted form $\mathrm{HH}(X / k, \alpha)$ of Hochschild homology as in the study of twisted $K$-theory. In fact, if $\alpha$ is the Brauer class of an Azumaya algebra $\mathcal{A}$, then $\mathrm{HH}(X / k, \alpha) \simeq \mathrm{HH}(\mathcal{A} / k)$. By work of Cortiñas-Weibel [14], there is a spectral sequence

$$
E_{2}^{s, t}=H^{s}\left(X, \Omega_{X / k}^{-t}\right) \Rightarrow H^{s+t}(\mathrm{HH}(X / k, \alpha)) .
$$

We call this the $\alpha$-twisted HKR spectral sequence; when $\alpha=0$, it is the HKR spectral sequence. In general, the terms of the $E_{2}$-page are the same as in the untwisted case, but the differentials might be different. When $k$ is a field and $X$ is additionally proper over $k$, the degeneration of the HKR spectral sequence is equivalent to the existence of an isomorphism as in (2).

Theorem 1.3. Let $k$ be a field of characteristic $p>0$. There exists a smooth projective threefold $X$ over $k$ and a Brauer class $\alpha \in \operatorname{Br}(X)$ such that the $\alpha$-twisted HKR spectral sequence does not degenerate. 
Specifically, we construct such an $X$ for which the differential $d_{2}^{\alpha}: H^{0}\left(X, \Omega_{X / k}^{0}\right) \rightarrow H^{2}\left(X, \Omega_{X / k}^{1}\right)$ in the $\alpha$-twisted HKR spectral sequence is nonzero.

For $p=2$, we can do a little better and find surface examples. In fact, classical Enriques surfaces work in that case (Propositions 7.7 and 9.2).

Theorem 1.3 gives examples of a smooth projective $k$-schemes $X$ and Azumaya algebras $\mathcal{A}$ on $X$ such that $\operatorname{dim}_{k} H^{i}(\mathrm{HH}(\mathcal{A} / k)) \neq \operatorname{dim}_{k} H^{i}(\mathrm{HH}(X / k))$. This is in contrast to the affine case of CortiñasWeibel [14] and shows that their theorem cannot be globalised. These examples also lead to cases of $\mathbf{P}^{n}$-bundles $P \rightarrow X$ such that pullback kills Hodge and de Rham cohomology classes, a phenomenon that can only exist in characteristic $p$. See Section 10.

Conventions. Throughout, we use cohomological indexing conventions. Given a commutative ring $k$, we will let $D(k)$ denote the derived $\infty$-category of $k$-modules and Sp denote the $\infty$-category of spectra. For the purposes of this article, a $d$-dimensional scheme is by definition equidimensional. Moreover, when we say that a spectral sequence discussed in Section 3 degenerates without specifying a page, the degeneration is always intended to begin at the first page where the spectral sequence is defined; when we assert that a spectral sequence does not degenerate, we specify the nonzero page and differential.

\section{Hochschild homology and de Rham cohomology of stacks}

Fix a commutative ring $k$. In this section, we introduce the various cohomology theories that we shall use later in the context of algebraic stacks over $k$. Our strategy is to define the cohomology of a stack via descent. To simplify definitions and avoid subtleties, we work with the syntomic topology and stick to stacks that are themselves syntomic (as defined next); this includes all smooth (or even local complete intersection) algebraic stacks over $k$, which is sufficient for our purposes.

Notation 2.1. A map $R \rightarrow S$ of commutative rings is called a syntomic map if it is flat and finitely presented with $L_{S / R} \in D(S)$ having Tor amplitude in $[-1,0]$; there is a similar definition for maps of schemes. Let $\operatorname{Syn}_{k}$ denote the category of syntomic $k$-algebras; its opposite category $\operatorname{Syn}_{k}^{o p}$, equipped with the Grothendieck topology where covers are given by finite families of syntomic maps that are jointly faithfully flat, is called the syntomic site of $k$. An algebraic stack $\mathcal{X} / k$ is called syntomic if there exists a syntomic cover $U \rightarrow \mathcal{X}$ with $U$ a syntomic $k$-scheme.

Example 2.2. Say $G / k$ is a flat and finitely presented affine group scheme. It is known that $G$ is a syntomic $k$-scheme. It follows that $B G$ is a syntomic $k$-stack: The canonical map $\operatorname{Spec}(k) \rightarrow B G$ is a syntomic cover because it is a $G$-torsor. In fact, $B G$ is actually a smooth $k$-stack: It suffices to check this fibrewise, and there it follows by realising $G$ as a closed subgroup scheme $G \hookrightarrow \mathrm{GL}_{n}$ and noting that the resulting map $\mathrm{GL}_{n} / G \rightarrow B G$ is a smooth surjection with a smooth source. Nevertheless, it is often more convenient in calculations to work with the syntomic cover $\operatorname{Spec}(k) \rightarrow B G$ (which is functorially defined in the group scheme $G$ ) rather than some noncanonical smooth atlas for $B G$.

Our goal is to give a definition of Hochschild and derived de Rham cohomologies (as well as variants) for syntomic $k$-stacks. Let us first recall the definitions of the relevant functors in the affine case; that is, as functors on $\operatorname{Syn}_{k}^{o p}$; we shall later extend these to all syntomic $k$-stacks via descent.

Definition 2.3. Fix $R \in \operatorname{Syn}_{k}$.

(a) For $i \geqslant 0$, we write $\wedge^{i} L_{R / k} \in D(R)$ for the $i$ th derived wedge power (in $R$-modules) of the cotangent complex $L_{R / k}$; these together form the Hodge cohomology of $R$.

(b) We let $\mathrm{HH}(R / k)=R \otimes_{R \otimes_{k} R}^{L} R \in D(k)$ denote the Hochschild homology of $R$ relative to $k$.

(c) The object $\mathrm{HH}(R / k)$ is equipped with a $k$-linear $S^{1}$-action, and we let $\mathrm{HC}^{-}(R / k)=\mathrm{HH}(R / k)^{h S^{1}} \in$ $D(k)$ denote the negative cyclic homology and $\operatorname{HP}(R / k)=\mathrm{HH}(R / k)^{t S^{1}} \in D(k)$ denote the periodic cyclic homology. 
(d) We let THH $(R) \in$ Sp denote the topological Hochschild homology of $R$, which is a spectrum (even an $E_{\infty}$-ring spectrum) with an $S^{1}$-action; we write $\operatorname{TP}(R)=\mathrm{THH}(R)^{t S^{1}}$ for its topological periodic cyclic homology. See [30] for a modern account of topological Hochschild homology and the structure on it. Recall also that if $k$ is a perfect field of characteristic $p$, then $\operatorname{TP}(R) / p \simeq \operatorname{HP}(R / k)$, as in [12, Theorem 6.7] or [6, Theorem 3.4]. Moreover, though $\operatorname{TP}(R)$ is typically a spectrum, if $R$ is an $\mathbf{F}_{p}$-algebra, $\operatorname{TP}(R)$ is naturally an object of $D\left(\mathbf{Z}_{p}\right)$.

(e) We let $L \Omega_{R / k}$ denote the derived de Rham complex of $R$, equipped with the derived Hodge filtration $\mathrm{Fil}_{H}^{\star} L \Omega_{R / k}=L \Omega_{R / k}^{\geqslant \star}$. Then $L \Omega_{R / k}^{\geqslant \star}$ is naturally an object of the filtered derived category $D F(k)$ (see $[12, \S 5]$ for more on the filtered derived category). By definition, if $R$ is a finitely generated polynomial ring over $k$, then $L \Omega_{R / k}^{\geqslant \star} \simeq \Omega_{R / k}^{\geqslant \star}$; that is, the derived de Rham complex with the derived Hodge filtration agrees with the ordinary de Rham complex and the filtration bête. We then define $L \Omega_{R / k}^{\geqslant \star}$ for general $R$ via left Kan extension. When $k$ has characteristic $p>0$ (which will be the case in our applications), it follows from [10] that $L \Omega_{R / k}^{\geqslant \star} \simeq \Omega_{R / k}^{\geqslant \star}$ for smooth $k$-algebras; in particular, $L \Omega_{R / k}$ is complete for the Hodge filtration for $R$ smooth.

Remark 2.4. Assume that $k$ has characteristic $p$ and is perfect. It was shown in [10, Theorem 3.27] that derived de Rham cohomology of syntomic algebras can be computed via crystalline cohomology; that is, for any syntomic $k$-algebra $R$, there is a natural isomorphism $L \Omega_{R / k} \simeq$ $R \Gamma_{\text {crys }}(\operatorname{Spec}(R) / k)$, with the Hodge filtration on $L \Omega_{R / k}$ matching up with the filtration coming from divided powers of the ideal sheaf on $R \Gamma_{\text {crys }}(\operatorname{Spec}(R) / k)$. Thus, this invariant admits a 'nonderived' definition. The derived definition is nevertheless useful because it is often easy to compute the cotangent complex and its derived exterior powers (especially once we extend to stacks).

(f) Suppose that $k$ is a perfect ring of characteristic $p$. In this situation, we write $R \Gamma_{\text {crys }}(\operatorname{Spec}(R)) \in$ $D(W(k))$ for the crystalline cohomology of $R$; because $k$ is perfect, we can take this to mean either absolute crystalline cohomology relative to the pd-base $\left(\mathbf{Z}_{p},(p)\right)$ or crystalline cohomology relative to the pd-base $(W(k),(p))$ without changing its meaning. By generalities on crystalline cohomology and Remark 2.4, we can regard $R \Gamma_{\text {crys }}(\operatorname{Spec}(R)) \in D(W(k))$ as a lift of $L \Omega_{R / k} \in D(k)$. We refer to $[12, \S 8]$ for a further discussion of this theory, including a description via the derived de Rham-Witt complex.

In order to extend these functors to syntomic $k$-stacks, we need the following descent result.

Theorem 2.5. The following assignments give sheaves on $\operatorname{Syn}_{k}^{o p}$ :

(1) the $D(k)$-valued functors $R \mapsto \wedge^{i} L_{R / k}$ (for all $\left.i \geqslant 0\right), H H(R / k), H C^{-}(R / k), H P(R / k)$;

(2) the $S p$-valued functors $R \mapsto T H H(R), T P(R)$;

(3) the $D(k)$-valued functor $R \mapsto L \Omega_{R / k}$ when $k$ has characteristic $p$;

(4) the $D(W(k))$-valued functor $R \mapsto R \Gamma_{\text {crys }}(\operatorname{Spec}(R))$ when $k$ is perfect of characteristic $p$.

In other words, given a syntomic cover $R \rightarrow R^{\prime}$, the natural maps

$$
F(R) \rightarrow \operatorname{Tot}\left(F\left(R^{\prime}\right) \Longrightarrow F\left(R^{\prime} \otimes_{R} R^{\prime}\right) \equiv \xi F\left(R^{\prime} \otimes_{R} R^{\prime} \otimes_{R} R^{\prime}\right) \equiv \xi \cdots\right)
$$

are equivalences for $F$ any of the above functors on $S_{y} n_{k}$.

Proof. We refer to [9, Remark 2.8] for the cotangent complex. In fact, [12, §3] covers all of the functors in (1) and (2), and [12, Example 5.12] covers (3); the claim in (4) follows formally from that in (3) because $R \Gamma_{\text {crys }}(\operatorname{Spec}(R))$ is derived $p$-complete (see [38, Tag 091N] for a treatment of this notion) and $R \Gamma_{\text {crys }}(\operatorname{Spec}(R)) / p \simeq L \Omega_{R / k}$.

Remark 2.6. The results of [12] are more general in that they show descent for stronger Grothendieck topologies (such as the quasisyntomic topology for all classes of functors above, and even the flat topology for the first two). These stronger results are critical to the methods of [12]. However, for the purpose of geometric applications in this article, the preceding generality suffices. 
Construction 2.7 (Cohomology of stacks). Fix a sheaf $F$ of spectra on $\operatorname{Syn}_{k}^{o p}$. ecause $F$ is a Zariski sheaf, we know how to make sense of $F(X)$ for any syntomic $k$-scheme $X$ by Zariski descent: We set $F(X)=R \Gamma\left(X_{Z a r}^{a f f}, F\right)$, where $X_{Z a r}^{a f f}$ denotes the category of affine opens in $X$ equipped with the usual topology. Similarly, if $\mathcal{X}$ is a syntomic $k$-stack, there is a tautological way to make sense of $F(\mathcal{X})$ by syntomic descent; that is, we set

$$
F(\mathcal{X}):=R \Gamma\left(\operatorname{Syn}_{k, / \mathcal{X}}^{o p}, F\right)
$$

In fact, we can be more explicit in practice: If $\mathcal{X}$ is a quasicompact syntomic $k$-stack with affine diagonal, then there exists an affine syntomic cover $U \rightarrow \mathcal{X}$ with $U$ a syntomic affine $k$-scheme, and Čech descent gives an identification

$$
R \Gamma\left(\operatorname{Syn}_{k, / \mathcal{X}}^{o p}, F\right) \simeq \operatorname{Tot}\left(F(U) \Longrightarrow F\left(U \times_{\mathcal{X}} U\right) \equiv F\left(U \times_{\mathcal{X}} U \times_{\mathcal{X}} U\right) \equiv\right.
$$

thus allowing one to compute the left side in terms of the value of $F$ on affines; a similar description applies to all syntomic $k$-stacks if one allows $U$ to be a possibly nonaffine syntomic $k$-scheme. Applying this construction to the functors from Theorem 2.5, we can obtain the following functors on syntomic $k$-stacks:

(1) the $D(k)$-valued functors $\mathcal{X} \mapsto R \Gamma\left(\mathcal{X}, \wedge^{i} L_{\mathcal{X} / k}\right)(\forall i), \operatorname{HH}(\mathcal{X} / k), \operatorname{HP}(\mathcal{X} / k)$;

(2) the Sp-valued functors $\mathcal{X} \mapsto \operatorname{THH}(\mathcal{X}), \operatorname{TP}(\mathcal{X})$;

(3) the $D(k)$-valued functor $\mathcal{X} \mapsto R \Gamma_{\mathrm{dR}}(\mathcal{X} / k)$ when $k$ has characteristic $p$;

(4) the $D(W(k))$-valued functor $\mathcal{X} \mapsto R \Gamma_{\text {crys }}(\mathcal{X})$ when $k$ is perfect of characteristic $p$.

The construction as a totalisation above also immediately makes it clear that if a sheaf $F$ has certain structural features when evaluated on syntomic $k$-schemes (respectively smooth $k$-schemes), it does so on syntomic $k$-stacks (respectively smooth $k$-stacks) as well. We shall implicitly exploit this observation later when extending certain natural filtrations on the invariants from Definition 2.3 to the stacky setting. Nonetheless, there are some subtleties.

Remark 2.8 (Comparison with the stack-theoretic cotangent complex). For a syntomic $k$-stack $\mathcal{X}$, one can show that $R \Gamma\left(\mathcal{X}, L_{\mathcal{X} / k}\right)$ as defined above is the global section of the stack-theoretic cotangent complex $L_{\mathcal{X} / k}$, considered as a quasicoherent complex on $\mathcal{X}$; this is the reason for the above notation. This follows from the transitivity triangle, which shows that the global sections of $L_{\mathfrak{X} / k}$ satisfy syntomic descent in $\mathfrak{X}$ (as in [9, Remark 2.8]).

Warning 2.9 (Comparison with Hochschild homology of perfect complexes). Fix a syntomic $k$-stack $\mathcal{X}$. The object $\mathrm{HH}(\mathcal{X} / k)$ constructed above does not (in general) coincide with $\operatorname{HH}(\operatorname{Perf}(\mathcal{X}) / k)$, the Hochschild homology of the $k$-linear stable $\infty$-category of perfect complexes on $\mathcal{X}$ : There is always a natural map $\mathrm{HH}(\operatorname{Perf}(\mathcal{X}) / k) \rightarrow \mathrm{HH}(\mathcal{X} / k)$, but it will not be an equivalence in general. For example, for $\mathcal{X}:=B \mathbf{G}_{m}$, one finds that both sides are concentrated in degree 0 where we obtain the completion map $k\left[t^{ \pm 1}\right] \rightarrow k \llbracket t-1 \rrbracket$ (cf. Example 4.3).

Warning 2.10 (HP versus the Tate construction). For any syntomic $k$-stack $\mathcal{X}$, the object $\mathrm{HH}(\mathcal{X} / k)$ inherits an $S^{1}$-action. It is always true that $\mathrm{HC}^{-}(\mathcal{X} / k)=\mathrm{HH}(\mathcal{X} / k)^{h S^{1}}$ because we can commute limits. However, $\operatorname{HP}(\mathcal{X} / k)$ may differ from $\operatorname{HH}(\mathcal{X} / k)^{t S^{1}}$ because the $S^{1}$-Tate construction does not generally commute with limits (compare Remark 4.16 below).

\section{Spectral sequences for stacks}

Continuing the notation of Section 2, we explain how the invariants introduced in Construction 2.7 come equipped with certain natural filtrations leading to spectral sequences. The differentials $d_{r}$ in our spectral sequences have bidegree $(r, 1-r)$. 
Definition 3.1. If a sheaf $F$ of spectra on $\operatorname{Syn}_{k}^{o p}$ is equipped with a complete descending $\mathbf{N}$-indexed filtration by sheaves, then its value on a syntomic $k$-stack $\mathcal{X}$ also admits a similar filtration. Applying this observation allows us to construct the following spectral sequences:

(a) Recall that for $R \in \operatorname{Syn}_{k}$, we have a complete descending $\mathbf{N}$-indexed multiplicative $S^{1}$-equivariant HKR filtration $\mathrm{Fil}_{\mathrm{HKR}}^{\star} \mathrm{HH}(R / k)$ with graded pieces $\operatorname{gr}_{\mathrm{HKR}}^{t} \mathrm{HH}(R / k) \simeq \wedge^{t} L_{R / k}[t]$, obtained by left Kan extending the Postnikov filtration on polynomial algebras. The HKR filtration on $\mathrm{HH}(-/ k)$ induces a complete descending $\mathbf{N}$-indexed filtration of $\operatorname{HH}(\mathcal{X} / k)$ with $\operatorname{gr}^{t}$ given by $R \Gamma\left(\mathcal{X}, \wedge^{t} L \mathcal{X} / k\right)[t]$. In particular, for any syntomic $k$-stack $\mathcal{X}$, we obtain the HKR spectral sequence

$$
E_{2}^{s, t}=H^{s}\left(\mathcal{X}, \wedge^{-t} L_{\mathcal{X} / k}\right) \Rightarrow H^{s+t}(\mathrm{HH}(\mathcal{X} / k))
$$

The HKR spectral sequence degenerates in characteristic 0 by [40].

(b) Assume that $\mathcal{X}$ is a smooth $k$-stack where $k$ has characteristic $p>0$. Restricting attention to smooth $k$-algebras and applying the reasoning used above, in conjunction with the last sentence of Definition 2.3(e), shows that the de Rham cohomology $R \Gamma_{\mathrm{dR}}(\mathcal{X} / k)$ admits a complete descending $\mathbf{N}$-indexed filtration $\mathrm{Fil}_{H}^{\star} R \Gamma_{\mathrm{dR}}(\mathcal{X} / k)$ with $\operatorname{gr}^{i}$ given by $R \Gamma\left(\mathcal{X}, \wedge^{i} L_{\mathcal{X} / k}\right)[-i]$. In particular, we obtain the Hodge-de Rham spectral sequence

$$
E_{1}^{s, t}=H^{t}\left(\mathcal{X}, \wedge^{s} L_{\mathcal{X} / k}\right) \Rightarrow H_{\mathrm{dR}}^{s+t}(\mathcal{X} / k)
$$

(b') Assume that $k$ is perfect of characteristic $p$. For any $k$-algebra $R$, the object $L \Omega_{R / k}$ comes endowed with a functorial increasing exhaustive $\mathbf{N}$-indexed filtration, called the conjugate filtration, with $\mathrm{gr}_{i}$ given by $\wedge^{i} L_{R^{(1)} / k}[-i]$ (see [10]). If one restricts attention to syntomic $k$-algebras, these graded pieces are coconnective. Because totalisations of cosimplicial coconnective objects commute with filtered colimits, we learn by descent that for any syntomic stack $\mathcal{X} / k$, we have a functorial increasing exhaustive $\mathbf{N}$-indexed filtration on $R \Gamma_{\mathrm{dR}}(\mathcal{X} / k)$ with $\operatorname{gr}_{i}$ given by $R \Gamma\left(\mathcal{X}, \wedge^{i} L_{\mathcal{X}^{(1)} / k}\right)[-i]$. In particular, we obtain the conjugate spectral sequence

$$
E_{2}^{s, t}=H^{s}\left(\mathcal{X}, \wedge^{t} L_{\mathcal{X}^{(1)} / k}\right) \Rightarrow H_{\mathrm{dR}}^{s+t}(\mathcal{X} / k)
$$

Remark 3.2. Comparing the $E_{2}$-terms of the conjugate spectral sequence with the $E_{1}$-terms of the Hodge-de Rham spectral sequence shows the following: If both the Hodge and de Rham cohomology groups of $\mathcal{X} / k$ are finite-dimensional in each degree, then the Hodge-de Rham spectral sequence degenerates if and only if the conjugate spectral sequence degenerates.

The preceding discussion also extends to $\mathbf{Z}$-indexed filtrations provided the graded pieces become highly coconnective for $i \rightarrow-\infty$. By the main results of [12], this yields the following two spectral sequences:

(c) Assume that $k$ has characteristic $p>0$ and $\mathcal{X}$ is a smooth $k$-stack. The motivic filtration on $\operatorname{HP}(-/ k)$ constructed in [12] (in the $p$-complete setting) and in general in [3] induces a complete exhaustive descending $\mathbf{Z}$-indexed filtration on $\operatorname{HP}(\mathcal{X} / k)$ with $\operatorname{gr}^{i}$ given by $R \Gamma_{\mathrm{dR}}(\mathcal{X} / k)$ [2i]. In particular, we obtain the de Rham-HP spectral sequence

$$
E_{2}^{s, t}=H_{\mathrm{dR}}^{s-t}(\mathcal{X} / k) \Rightarrow H^{s+t}(\mathrm{HP}(\mathcal{X} / k))
$$

There is a variant for $\mathrm{HC}^{-}(\mathcal{X} / k)$ : One has a complete exhaustive $\mathbf{Z}$-indexed descending filtration on $\mathrm{HC}^{-}(\mathcal{X} / k)$ with $\operatorname{gr}^{t}$ given by $\mathrm{Fil}_{H}^{t} R \Gamma_{\mathrm{dR}}(\mathcal{X} / k)[2 t]$ and a similar spectral sequence.

(d) Assume that $k$ is a perfect ring of characteristic $p$ and $\mathcal{X}$ is a smooth $k$-stack. The motivic filtration on $\operatorname{TP}(-)$ (cf. [12]) induces a complete exhaustive descending $\mathbf{Z}$-indexed filtration on $\operatorname{TP}(\mathcal{X})$ with $\mathrm{gr}^{i}$ given by $R \Gamma_{\text {crys }}(\mathcal{X})[2 i]$. In particular, we obtain the crystalline version of the de Rham-HP 
spectral sequence, namely, the crystalline-TP spectral sequence

$$
E_{2}^{s, t}=H_{\text {crys }}^{s-t}(\mathcal{X}) \Rightarrow H^{s+t}\left(\mathrm{TP}\left(\mathcal{X} ; \mathbf{Z}_{p}\right)\right)
$$

the target is the $p$-completion of topological periodic cyclic homology.

(e) For any syntomic $k$-stack $\mathcal{X}$, we have the Tate spectral sequence

$$
E_{2}^{s, t}=H_{\text {Tate }}^{s}\left(B S^{1}, H^{t}(\mathrm{HH}(\mathcal{X} / k))\right) \Rightarrow H^{s+t}\left((\mathrm{HH}(\mathcal{X} / k))^{t S^{1}}\right) .
$$

If $\mathcal{X}$ is a syntomic $k$-scheme, then we can identify $\operatorname{HH}(\mathcal{X} / k)^{t S^{1}}$ with $\operatorname{HP}(\mathcal{X} / k)$.

Remark 3.3. The smoothness assumptions were made in Definition 3.1 to ensure that derived de Rham cohomology is complete for the Hodge filtration. We could drop this assumption entirely if we replaced derived de Rham cohomology with its Hodge-completed variant (and derived crystalline cohomology with its Nygaard completed variant). However, because the stacks that we shall encounter later are smooth, we prefer to stick to the limited generality introduced above.

We organise the spectral sequences introduced in Figure 1, borrowed from [4].

Remark 3.4. The Hodge-de Rham spectral sequence degenerates in characteristic 0 for smooth proper schemes by Hodge theory. Moreover, if $k$ is a perfect field of characteristic $p$ and $X / k$ is a smooth proper $k$-scheme with $\operatorname{dim}(X) \leqslant p$ that lifts to $W_{2}(k)$, then the Hodge-de Rham spectral sequence degenerates by Deligne-Illusie [17]. Remarkably, it is still unknown whether the hypothesis on dimension is necessary in the preceding statement: Could it be true that the Hodge-de Rham spectral sequence degenerates for any smooth proper scheme over a perfect field $k$ of characteristic $p$ that is liftable to $W_{2}(k)$ (or even $W(k))$ ? This question was explicitly raised in [23, Problem 7.10], and Deligne-Illusie presumed that the answer is 'no'.

Remark 3.5 (The noncommutative Tate spectral sequence). In Definition 3.1, the first four spectral sequences crucially use algebraic geometry. However, the Tate spectral sequence extends to the noncommutative setting: For any $k$-linear stable $\infty$-category $\mathcal{C}$, there is a spectral sequence

$$
E_{2}^{s, t}=H_{\text {Tate }}^{s}\left(B S^{1}, H^{t}(\mathrm{HH}(\mathcal{C} / k))\right) \Rightarrow H^{s+t}(\mathrm{HP}(\mathcal{C} / k)) .
$$

In this context, the Tate spectral sequence is also called the noncommutative Hodge-de Rham spectral sequence. Let $k$ be a perfect field of characteristic $p$. A result of Kaledin [25, 26] (see also [29]) implies that if $\mathcal{C}$ is a smooth proper $k$-linear stable $\infty$-category (such as $\operatorname{Perf}(X)$ where $X$ is a smooth proper $k$-scheme) such that $H^{i}(\mathrm{HH}(\mathcal{C} / k))=0$ for $i \notin[-p, p]$ (the noncommutative analogue of $\operatorname{dim}(X) \leqslant p$ ) and if $\mathcal{C}$ lifts to $W_{2}(k)$, then the Tate spectral sequence degenerates at $E_{2}$. Kaledin used this to prove that the Tate spectral sequence degenerates for smooth proper dg categories over characteristic 0 fields, which together with HKR in characteristic 0 implies Hodge-de Rham degeneration.

Remark 3.6 (A degeneration criterion). Suppose that $X$ is a smooth and proper variety over a perfect field $k$ of characteristic $p$. In this case, all of the $k$-vector spaces appearing in Figure 1 are finite-dimensional.

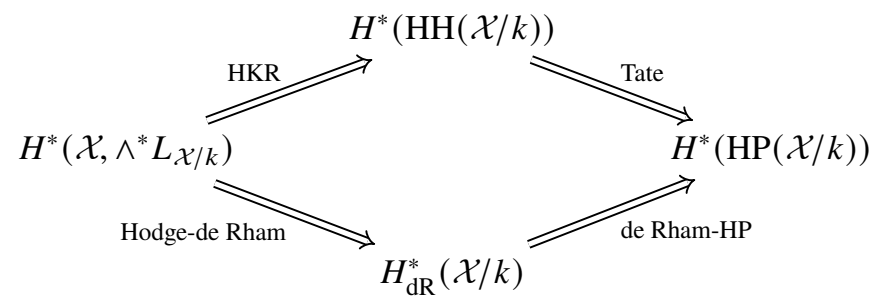

Figure 1. The Hodge quartet. 
Thus, we can use dimension counts to make conclusions about degeneration of the spectral sequences. For example, if $\operatorname{dim} X \leqslant p$, then the HKR spectral sequence degenerates [7]. This implies that the Tate spectral sequence degenerates if and only if both the Hodge-de Rham and de Rham-HP spectral sequences degenerate. In particular, if the Hodge-de Rham spectral sequence does not degenerate, then neither does the Tate spectral sequence.

The literature (see, for example, [17, Remarques 2.6(i)]) provides many examples of smooth proper surfaces where the Hodge-de Rham spectral sequence, and hence the Tate spectral sequence, does not degenerate. In this article, we provide examples that witness the nondegeneration of the remaining spectral sequences; that is, the HKR, de Rham-HP, and crystalline-TP spectral sequences.

\section{Classifying space counterexamples}

In this section, we establish counterexamples to degeneration of the HKR spectral sequence for certain algebraic stacks. Our examples are the classifying stacks of finite flat group schemes. In fact, the group schemes $\mu_{p}, \mu_{p} \times \mu_{p}$ and $\alpha_{p}$ already lead to the desired counterexamples. ${ }^{1}$ Later, we will approximate, in the sense of Totaro [41], these classifying stacks by smooth projective $k$-schemes to prove Theorem 1.1.

In this section, we work over a fixed perfect field $k$ of characteristic $p>0$. For a finite flat $k$-group scheme $G$, we will let $B G$ denote the classifying stack of $G$-torsors for the fppf-topology. Note that these stacks are always smooth (Example 2.2).

Our arguments begin with the calculation of the Hodge cohomology of $B G$ and are phrased in terms of the co-Lie complex co-Lie $(G) \in D(B G)$ of [21, Ch. VII, 3.1.2]. This is a $G$-equivariant refinement of $e^{*} L_{G / k}$ for $e: \operatorname{Spec}(k) \rightarrow G$ the identity section and can be identified with the cotangent complex of the stack $B G$ up to a shift. When $G$ is smooth, co- $\operatorname{Lie}(G)$ is the linear dual of the adjoint representation of $G$. The main technical tool is the following result.

Theorem 4.1 (Totaro, cf. [42, Theorem 3.1]). There is a multiplicative, graded isomorphism

$$
R \Gamma\left(B G, \bigoplus_{i \geqslant 0} \wedge^{i} L_{B G / k}\right) \simeq R \Gamma\left(G, \bigoplus_{i \geqslant 0} \operatorname{Sym}^{i}(\cos \operatorname{Lie}(G))[-i]\right)
$$

in $D(k)$, where the right side denotes 'rational cohomology' of $G$-representations.

The result comes from the fact (which to formulate we use the cotangent complex of stacks) that if $\pi: \operatorname{Spec}(k) \rightarrow B G$ denotes the tautological map, then there is a multiplicative, graded, $G$-equivariant isomorphism

$$
\pi^{*}\left(\bigoplus_{i \geqslant 0} \wedge^{i} L_{B G / k}\right) \simeq \bigoplus_{i \geqslant 0} \operatorname{Sym}^{i}(\operatorname{co}-\operatorname{Lie}(G))[-i],
$$

which implies the isomorphism (3) in $D(k)$.

In our examples, the basic source for nondegeneration is the following. The Frobenius on the classifying stacks $B \mu_{p}, B \alpha_{p}$ factors through a point, which forces the Frobenius to act by zero on the zeroth Hochschild homology. However, the $p$ th power map is not zero in the relevant Hodge cohomology (in fact, the $p$ th power map does not agree with the map induced by the Frobenius), which forces the existence of differentials in the HKR spectral sequence. Another phenomenon that leads to nondegeneration of the Hodge-to-de Rham spectral sequence for $B \alpha_{p}$ is its failure to lift to $W_{2}(k)$, which leads to the nondegeneration of the conjugate spectral sequence.

\footnotetext{
${ }^{1}$ These examples exhibit qualitatively different behaviour: The group schemes $\mu_{p}$ or $\mu_{p} \times \mu_{p}$ lift to $W_{2}(k)$ compatibly with Frobenius and their Hodge-de Rham spectral sequences always degenerate (Proposition 4.6), whereas the group scheme $\alpha_{p}$ does not lift to $W_{2}(k)$ and its Hodge-de Rham spectral sequence does not degenerate (Proposition 4.12 or Remark 4.13). Nevertheless, either example leads to a nondegenerate HKR spectral sequence.
} 


\section{1. $B \mu_{p}$}

In this section, we analyze the spectral sequences for $B \mu_{p}$. Let us begin with a (special case of much more general) degeneration criterion for the Hodge-de Rham spectral sequence.

Lemma 4.2. Let $\mathcal{X}=B G$ for a diagonalisable group scheme $G$ (such as $\mu_{p}$ or $\left.\boldsymbol{G}_{m}\right)$. Then the conjugate and Hodge-de Rham spectral sequences for $\mathcal{X}$ degenerate.

Proof. Using [17, Remark 2.2 (ii)] as well as functorial simplicial resolutions, one shows that for any syntomic $k$-algebra $R$ equipped with a lift $\tilde{R}$ to $W_{2}(k)$ together with a lift $\tilde{\phi}: \tilde{R} \rightarrow \tilde{R}$ of the Frobenius, there is a functorial (in the pair $(\tilde{R}, \tilde{\phi})$ ) isomorphism $\oplus_{i \geqslant 0} \wedge^{i} L_{R^{(1)} / k}[-i] \simeq L \Omega_{R / k}$; that is, the conjugate filtration splits functorially in the lifting data. Now the syntomic hypercover of $\mathcal{X}=B G$ given by the Čech nerve of $\operatorname{Spec}(k) \rightarrow B G$ is a simplicial syntomic affine $k$-scheme that comes equipped with such lifting data compatibly with the simplicial structure maps. Applying the preceding splitting levelwise and totalising gives an isomorphism $R \Gamma_{\mathrm{dR}}(B G / k) \simeq \oplus_{i \geqslant 0} R \Gamma\left(B G, \wedge^{i} L_{B G / k}[-i]\right)$; that is, the conjugate filtration splits and thus the conjugate spectral sequence degenerates. Dimension considerations now show that the Hodge-de Rham spectral sequence must also degenerate.

This allows us to recover the following standard calculation. As usual, let $P(c)$ denote a polynomial ring over $k$ on the generator $c$ and let $E(d)$ denote an exterior algebra over $k$ on the generator $d$.

Example 4.3 (Cohomology of $\left.B \mathbf{G}_{m}\right)$. We have that co-Lie $\left(\mathbf{G}_{m}\right)$ is the trivial representation of $\mathbf{G}_{m}$. Because $R \Gamma\left(B \mathbf{G}_{m}, \mathcal{O}\right) \simeq k$, it follows that $H^{*}\left(B \mathbf{G}_{m}, \wedge^{*} L_{B \mathbf{G}_{m} / k}\right) \simeq P(c)$, where $c$ has bidegree $(1,1)$. By Lemma 4.2, we also have $H_{\mathrm{dR}}^{*}\left(B \mathbf{G}_{m}\right) \simeq P(c)$. The generator $c$ can be explicitly chosen as the first Chern class of the tautological line bundle on $B \mathbf{G}_{m}$. The HKR spectral sequence for $H^{*}\left(\mathrm{HH}\left(B \mathbf{G}_{m} / k\right)\right)$ degenerates and we find that $H^{*}\left(\operatorname{HH}\left(B \mathbf{G}_{m} / k\right)\right) \simeq k[[u]]$ for a class $u$ in degree zero. In fact, all of the spectral sequences considered above degenerate for $B \mathbf{G}_{m}$ because there is no room for differentials.

Let us first review the Hodge and de Rham cohomologies of $B \mu_{p}$. Part (i) of the following proposition is a consequence of the fact that co- $\operatorname{Lie}\left(\mu_{p}\right) \simeq \mathcal{O} \oplus \mathcal{O}[1] \in D\left(\mu_{p}\right)$, which yields the calculation of Hodge cohomology. Part (ii) follows from the fact that the group scheme $\mu_{p}$ lifts to characteristic 0 with a lift of Frobenius.

Proposition 4.4 (cf. [42, Proposition 10.1]).

(i) The Hodge cohomology of $B \mu_{p}$ is given by

$$
H^{*}\left(B \mu_{p}, \wedge^{*} L_{B \mu_{p} / k}\right) \cong E(d) \otimes P(c),
$$

where $d \in H^{0}\left(B \mu_{p}, L_{B \mu_{p} / k}\right)$ and $c \in H^{1}\left(B \mu_{p}, L_{B \mu_{p} / k}\right)$.

(ii) The Hodge-de Rham and conjugate spectral sequences degenerate for $B \mu_{p}$ and we have an isomorphism

$$
H_{d R}^{*}\left(B \mu_{p} / k\right) \cong E(d) \otimes P(c),
$$

where $|d|=1$ and $|c|=2$.

Proposition 4.5. The Hochschild homology ring $H^{*}\left(H H\left(B \mu_{p} / k\right)\right)$ is isomorphic to $k[c] /\left(c^{p}\right)$, where $|c|=0$. In particular, it is concentrated in degree 0 .

Proof. We use the HKR spectral sequence to calculate $H^{*}\left(\mathrm{HH}\left(B \mu_{p} / k\right)\right)$. Its $E_{2}$-page is calculated using Proposition 4.4. Using the notation there, one sees that for degree reasons, the class $c \in H^{1}\left(B \mu_{p}, L_{B \mu_{p} / k}\right)$ must be permanent. Thus, it defines a nonzero class of $H^{0}\left(\mathrm{HH}\left(B \mu_{p} / k\right)\right)$ that we also call $c$. Note that $c \in H^{0}\left(\mathrm{HH}\left(B \mu_{p} / k\right)\right)$ is annihilated by the pullback along the tautological map $\operatorname{Spec}(k) \rightarrow B \mu_{p}$. On the other hand, the Frobenius $\varphi$ on $B \mu_{p}$ factors through this map, so we see 
that $\varphi(c)=0$ in $H^{0}\left(\mathrm{HH}\left(B \mu_{p} / k\right)\right)$. But $\varphi$ acts on $H^{0}\left(\mathrm{HH}\left(B \mu_{p} / k\right)\right)$ by the $p$-power map, ${ }^{2}$ so multiplicativity of the HKR spectral sequence shows that $\varphi(c)$ is represented by $c^{p} \in H^{p}\left(B \mu_{p}, \wedge^{p} L_{B \mu_{p} / k}\right)$, whence the latter must be a boundary in the HKR spectral sequence. To proceed further, observe that we have a filtered map $\mathrm{HH}\left(B \mu_{p} / k\right) \rightarrow \mathrm{HH}\left(B \mu_{p} / k\right) / \mathrm{Fil}_{\mathrm{HKR}}^{p}$ as well as a formality isomorphism $\mathrm{HH}\left(B \mu_{p} / k\right) / \mathrm{Fil}_{\mathrm{HKR}}^{p} \simeq \oplus_{i \leqslant p-1} R \Gamma\left(B \mu_{p}, \wedge^{i} L_{B \mu_{p} / k}[i]\right)$ in $D F(k)$. By contemplating the induced map on spectral sequences, we learn that the only differential that can possibly hit $c^{p} \in H^{p}\left(B \mu_{p}, \wedge^{p} L_{B \mu_{p} / k}\right)$ in the HKR spectral sequence of $B \mu_{p}$ is $d_{p}(d)$ (up to a unit). This completes the proof because now on the $E_{p+1}$-page of the spectral sequence we are left only with the nonzero classes $1, c, \ldots, c^{p-1}$.

Theorem 4.6. For $B \mu_{p}$, the following assertions hold:

(1) The HKR spectral sequence does not degenerate. There is a nonzero differential $d_{p}: H^{0}\left(B \mu_{p}, L_{B \mu_{p} / k}\right) \rightarrow H^{p}\left(B \mu_{p}, \wedge^{p} L_{B \mu_{p} / k}\right)$.

(2) The Hodge-de Rham and conjugate spectral sequences degenerate.

(3) The Tate spectral sequence for $\mathrm{HH}\left(B \mu_{p} / k\right)^{t S^{1}}$ degenerates.

(4) The de Rham-HP spectral sequence does not degenerate. There is a nonzero differential $d_{p}: H_{d R}^{1}\left(B \mu_{p} / k\right) \rightarrow H_{d R}^{2 p}\left(B \mu_{p} / k\right)$.

(5) The crystalline-TP spectral sequence degenerates, but the resulting filtration on $H^{*}\left(T P\left(B \mu_{p}\right)\right)$ is not split.

Proof.

(1) This was shown in the course of the proof of Proposition 4.5.

(2) This was shown in Proposition 4.4 (see also Lemma 4.2).

(3) This follows for degree reasons because $\mathrm{HH}\left(B \mu_{p} / k\right)$ is concentrated in degree 0 .

(4) It follows from the calculation of $\mathrm{HH}\left(B \mu_{p} / k\right)$ that $H^{*}\left(\mathrm{HC}^{-}\left(B \mu_{p} / k\right)\right) \cong P(t) \otimes_{k} k[c] /\left(c^{p}\right)$, where $|t|=2$. In particular, this theory is concentrated in even degrees. Because we know that $B \mu_{p}$ has de Rham cohomology in odd degree (Proposition 4.4), the spectral sequence from Hodgefiltered de Rham cohomology to $\mathrm{HC}^{-}$cannot degenerate. By naturality, it follows that the de Rham-HP spectral sequence cannot degenerate either. Explicitly, we find that the de Rham-HPspectral sequence has $E_{2}$-term given by $E(d) \otimes P(c) \otimes P\left(t^{ \pm 1}\right)$; here $t$ is a permanent cycle because it comes from the cohomology of $S^{1}$ and $c$ is a permanent cycle because it comes from $B \mathbf{G}_{m}$. By the description of $H^{*}\left(\mathrm{HC}^{-}\left(B \mu_{p} / k\right)\right)$, it follows that $c^{p}=0$, so we must have a nonzero differential $d_{p}: H_{\mathrm{dR}}^{1}\left(B \mu_{p} / k\right) \rightarrow H_{\mathrm{dR}}^{2 p}\left(B \mu_{p} / k\right)$ annihilating $c^{p}$. The spectral sequence now shows that $H^{*}\left(\mathrm{HP}_{*}\left(B \mu_{p} / k\right)\right) \simeq P\left(t^{ \pm 1}\right) \otimes k[c] / c^{p}$, where $|t|=2$.

(5) Because $\operatorname{TP}\left(B \mu_{p}\right) / p \simeq \operatorname{HP}\left(B \mu_{p} / k\right)$, the calculation in (4) implies that $H^{*}\left(\operatorname{TP}\left(B \mu_{p}\right)\right)$ is concentrated in even degrees and $p$-torsion free. On the other hand, we have

$$
H_{\text {crys }}^{*}\left(B \mu_{p}\right) \simeq W[c] /(p c),
$$

where $|c|=2$ : This follows from the isomorphism $R \Gamma_{\text {crys }}\left(B \mu_{p}\right) / p \simeq R \Gamma_{\mathrm{dR}}\left(B \mu_{p} / k\right)$, the calculation in Proposition 4.4 and the observation that multiplication by $n$ on $H_{\text {crys }}^{2}\left(B \mu_{p}\right)$ is induced by the multiplication by $n$ endomorphism of $B \mu_{p}$ (and is thus the 0 map when $p \mid n$ ). It follows that all terms on the $E_{2}$-page of the crystalline-TP spectral sequence are in even degrees, so the spectral sequence degenerates. Because $H_{\text {crys }}^{*}\left(B \mu_{p}\right)$ contains nonzero $p$-torsion elements while $H^{*}\left(\operatorname{TP}\left(B \mu_{p}\right)\right)$ is $p$ torsion free, the filtration on $H^{*}\left(\operatorname{TP}\left(B \mu_{p}\right)\right)$ coming from this spectral sequence cannot split.

Remark 4.7 (The HKR filtration does not split for Frobenius lifts). In analogy with Deligne-Illusie [17, Remark 2.2 (ii)], one might wonder the following: Given a smooth $k$-algebra with a lift $\tilde{R}$ to $W_{2}(k)$ and

\footnotetext{
${ }^{2}$ Given an $\mathbf{F}_{p}$-algebra $R$, the endomorphism of $\mathrm{HH}\left(R / \mathbf{F}_{p}\right)$ induced by the Frobenius on $R$ coincides with the Frobenius endomorphism of the simplicial commutative $\mathbf{F}_{p}$-algebra $\mathrm{HH}\left(R / \mathbf{F}_{p}\right)$. Applying this observation to a hypercover shows that for any algebraic stack $\mathcal{X} / \mathbf{F}_{p}$, the endomorphism of $\boldsymbol{H}^{0}\left(\mathrm{HH}\left(\mathcal{X} / \mathbf{F}_{p}\right)\right)$ induced by the Frobenius on $\mathcal{X}$ is the $p$-power map.
} 
a lift $\tilde{\phi}: \tilde{R} \rightarrow \tilde{R}$ of the Frobenius, can one choose an isomorphism $\mathrm{HH}(R / k) \simeq \oplus_{i} \Omega_{R / k}^{i}[i]$ splitting the HKR filtration that is functorial in the lifting data $(\tilde{R}, \tilde{\phi})$ ? Theorem $4.6(1)$ shows that this is not possible (via the argument of Lemma 4.2 to pass from the affine case to stacks).

Finally, let us use the calculations above to record an example where the crystalline-TP spectral sequence does not degenerate.

Lemma 4.8. The crystalline-TP spectral sequence for $B\left(\mu_{p} \times \mu_{p}\right)$ does not degenerate.

Proof. We saw in the proof of (3) of Theorem 4.6, via the de Rham-HP spectral sequence, that $H^{*}\left(\operatorname{HP}\left(B \mu_{p} / k\right)\right)$ is concentrated in even degrees and given by $P\left(t^{ \pm 1}\right) \otimes_{k} k[c] / c^{p}$. Though HP does not in general satisfy a Künneth formula for syntomic $k$-stacks, de Rham cohomology does. Running the de Rham-HP spectral sequence again, we find that $H^{*}\left(\operatorname{HP}\left(B\left(\mu_{p} \times \mu_{p}\right) / k\right)\right)$ is concentrated in even degrees. Therefore, $H^{*}\left(\operatorname{TP}\left(B\left(\mu_{p} \times \mu_{p}\right)\right)\right)$ is concentrated in even degrees and is $p$-torsion free, because $\mathrm{TP} / p \simeq \mathrm{HP}$. On the other hand, $H_{\text {crys }}^{3}\left(B\left(\mu_{p} \times \mu_{p}\right)\right) \cong k$ by Künneth. The lemma follows.

\section{2. $B \alpha_{p}$}

In this subsection, we calculate everything explicitly for $B \alpha_{p}$. We shall crucially exploit the natural $\mathbf{G}_{m}$-action on $B \alpha_{p}$, induced (ultimately) from the $\mathbf{G}_{m}$-action on $\mathbf{G}_{a}$, defined formally as follows.

Observation 4.9 (Gradings). The group scheme $\alpha_{p}=\operatorname{Spec}\left(k[t] /\left(t^{p}\right)\right)$ has a natural $\mathbf{G}_{m}$-action defined by requiring the function $t$ to have weight 1 . This induces a $\mathbf{G}_{m}$-action on $B \alpha_{p}$ and, consequently, there is a natural weight grading on associated cohomological invariants, such as Hodge, Hochschild and de Rham cohomologies. Moreover, the differentials in the relevant spectral sequences respect the weight grading.

Proposition 4.10. If $p>2$, the Hodge cohomology of $B \alpha_{p}$ is given by

$$
H^{*}\left(B \alpha_{p}, \wedge^{*} L_{B \alpha_{p} / k}\right) \cong E(\alpha) \otimes P(\beta) \otimes E(s) \otimes P(u),
$$

where $\alpha \in H^{1}\left(B \alpha_{p}, \mathcal{O}\right), \beta \in H^{2}\left(B \alpha_{p}, \mathcal{O}\right), s \in H^{0}\left(B \alpha_{p}, L_{B \alpha_{p} / k}\right)$ and $u \in H^{1}\left(B \alpha_{p}, L_{B \alpha_{p} / k}\right)$. Moreover, the weights of $\alpha, \beta, s$ and $u$ are $1, p, p$ and 1 , respectively. For $p=2$, we replace $E(\alpha) \otimes P(\beta)$ with $P(\alpha)$.

Proof. We begin by showing that $H^{*}\left(B \alpha_{p}, \mathcal{O}\right) \simeq E(\alpha) \otimes P(\beta)$ with degrees and weights as in the proposition. By Cartier duality, ${ }^{3}$ we have $H^{*}\left(B \alpha_{p}, \mathcal{O}\right) \simeq \operatorname{Ext}_{k[s] /\left(s^{p}\right)}^{*}(k, k)$, where $k[s] /\left(s^{p}\right)$ denotes the Hopf algebra of functions on the Cartier dual of $\alpha_{p}$ (and is thus also a copy of $\alpha_{p}$ itself, but the weight of the generator $s$ is now -1). One then calculates using the standard resolution

$$
\left(\cdots k[s] /\left(s^{p}\right) \stackrel{s^{p-1}}{\longrightarrow} k[s] /\left(s^{p}\right) \stackrel{s}{\rightarrow} k[s] /\left(s^{p}\right) \stackrel{s^{p-1}}{\longrightarrow} k[s] /\left(s^{p}\right) \stackrel{s}{\rightarrow} k[s] /\left(s^{p}\right)\right) \stackrel{\text { can }}{\simeq} k,
$$

graded in a natural way, that the answer is as predicted. Alternately, one can find this calculation in [19, Theorem 2.4].

To compute Hodge cohomology, we first calculate the co-Lie complex. Using the closed immersion $\alpha_{p} \subset \mathbf{G}_{a}$ of group schemes, we learn that $L_{\alpha_{p} / k}$ is computed by the 2-term complex $\left(t^{p}\right) /\left(t^{2 p}\right) \stackrel{d}{\rightarrow} k[t] /\left(t^{p}\right) d t$. Restricting along the origin gives a 2-term complex of $\alpha_{p}$-representations computing co-Lie $\left(\alpha_{p}\right)$ as an object of $D(k)$. Using this complex, we find that $H^{0}\left(\operatorname{co}-\operatorname{Lie}\left(\alpha_{p}\right)\right)=$

${ }^{3}$ For a finite $k$-group scheme $G$, the abelian category of coherent sheaves $\operatorname{Coh}(B G)$ on $B G$ can be identified as the category $\operatorname{Rep}^{f}(G)$ of finite-dimensional representations $G$; that is, as the category $\operatorname{CoMod}_{\mathcal{O}(G)}^{f}$ of finite dimensional comodules over the $k$-coalgebra $\mathcal{O}(G)$. When $G$ is commutative, this is anti-equivalent to the category $\operatorname{Mod}_{\mathcal{O}\left(G^{\vee}\right)}^{f}$ of finite-dimensional modules over $k$-algebra $\mathcal{O}(G)^{\vee} \simeq \mathcal{O}\left(G^{\vee}\right)$, where $G^{\vee}$ denotes the Cartier dual of $G$. Under this identification, the trivial representation of $G$ corresponds to the residue field at the origin on $G^{\vee}$. Computing Ext-groups now gives the isomorphism used above. 
$H^{-1}\left(\operatorname{co}-\operatorname{Lie}\left(\alpha_{p}\right)\right)=k$ are both the trivial one-dimensional representation of $\alpha_{p}$. Furthermore, $H^{0}$ (corresponding to $d t$ ) is concentrated in weight 1 and $H^{-1}$ (corresponding to $t^{p}$ ) is concentrated in weight $p$. But then co-Lie $\left(\alpha_{p}\right) \in D\left(B \alpha_{p}\right)$ splits as $\mathcal{O} \oplus \mathcal{O}[1]$ : The obstruction to splitting is a weight $p-1$ map $\mathcal{O} \rightarrow \mathcal{O}[2]$, and there are no such maps by the calculation of $H^{2}\left(B \alpha_{p}, \mathcal{O}\right)$ explained in the previous paragraph. Another approach to seeing this description of co-Lie $\left(\alpha_{p}\right)$ is to use that $\alpha_{p}$ is commutative and [21, Ch. VII, Prop. 4.1.1].

Thus, we learn that, as objects in $D\left(B \alpha_{p}\right)$, we have co-Lie $\left(\alpha_{p}\right) \simeq \mathcal{O} s[1] \oplus \mathcal{O} u$, where $s$ corresponds to a class in $H^{0}\left(B \alpha_{p}, L_{B \alpha_{p} / k}\right)$, which has weight $p$, and $u$ corresponds to a class $H^{1}\left(B \alpha_{p}, L_{B \alpha_{p} / k}\right)$, which has weight 1 . As in Proposition 4.4, one then finds that

$$
\bigoplus_{i \geqslant 0} \operatorname{Sym}^{i}(\operatorname{co}-\operatorname{Lie}(G))[-i] \simeq E(s) \otimes P(u) \otimes \mathcal{O} \in D\left(B \alpha_{p}\right)
$$

Combining this with the calculation of $H^{*}\left(B \alpha_{p}, \mathcal{O}\right)$ and using the projection formula then gives the desired answer.

For the next result, we recall that $\mathrm{HH}\left(B \alpha_{p} / k\right)$ acquires an action of the circle $S^{1}$, inducing an operator $H^{*}\left(\mathrm{HH}\left(B \alpha_{p} / k\right)\right) \rightarrow H^{*-1}\left(\mathrm{HH}\left(B \alpha_{p} / k\right)\right)$ given by multiplication by the fundamental class of $S^{1}$; this is also identified (up to 2-periodicity) with the first differential in the Tate spectral sequence.

Proposition 4.11. If $p$ is odd, then $H^{*}\left(H H\left(B \alpha_{p} / k\right)\right)$ is isomorphic to $E(\alpha) \otimes P(\beta) \otimes k[u] / u^{p}$ with $\alpha$ having degree 1 and weight $1, \beta$ having degree 2 and weight $p$ and $u$ having degree 0 and weight 1 . If $p=2$, then $H^{*}\left(H H\left(B \alpha_{p} / k\right)\right)$ is given by $k[\alpha] \otimes k[u] / u^{p}$ if $p=2$ with the same degrees and weights as in the odd case. The circle action carries $\alpha \mapsto u$ (up to units); in particular, the Tate spectral sequence for $H H\left(B \alpha_{p} / k\right)^{t S^{1}}$ does not degenerate.

Proof. We give the proof when $p$ is odd. We will use the HKR spectral sequence and the calculation of the $E_{2}$-page coming from Proposition 4.10. Note that $|\alpha|=(1,0),|\beta|=(2,0),|s|=(0,-1)$ and $|u|=(1,-1)$ in the $E_{2}$-page of the HKR spectral sequence.

We begin by noting that $\alpha$ and $\beta$ are permanent cycles arising from the map $R \Gamma\left(B \alpha_{p}, \mathcal{O}\right) \rightarrow$ $\mathrm{HH}\left(B \alpha_{p} / k\right)$ that comes from choosing a base point on $S^{1}$. (In fact, we recall that $\mathrm{HH}(\mathcal{X} / k)$ must contain $R \Gamma(\mathcal{X}, \mathcal{O})$ as a summand for any syntomic stack $\mathcal{X} / k$.) Next, because every differential respects the weight grading, we conclude that $u$ (which has weight 1 ) is a permanent cycle: All weights that occur on the target of a differential emanating from $u$ have weights $>1$. Finally, we claim $d_{p}(s)=u^{p}$ up to units: This is proven like the analogous claim in Proposition 4.5 , noting that Frobenius on $B \alpha_{p}$ factors through a point. There are no further differentials (because $\alpha, \beta$ and $u$ are permanent), so we obtain that $H^{*}\left(\mathrm{HH}\left(B \alpha_{p} / k\right)\right)$ has the predicted shape.

For the circle action, we use the following observation: If $R$ is any nonnegatively graded commutative $k$-algebra with $R_{0}=k$, then we have an $S^{1}$-equivariant equivalence in weight $1, \mathrm{HH}(R / k)_{\mathrm{wt}=1} \simeq$ $C_{*}\left(S^{1} ; k\right) \otimes_{k}\left(L_{R / k}\right)_{\mathrm{wt}=1}$ as one sees by reducing to the free case. In particular, in weight 1 , the circle action on $\mathrm{HH}(-/ k)$ is always induced. Because this is functorial, it applies to $B \alpha_{p}$, too, and we find that $H^{*}\left(\mathrm{HH}\left(B \alpha_{p} / k\right)\right)$ in weight 1 has an induced $S^{1}$-action, whence the claim.

Proposition 4.12. For all $p$, the de Rham cohomology of $B \alpha_{p}$ is given by

$$
H_{d R}^{*}\left(B \alpha_{p} / k\right) \simeq E\left(\alpha^{\prime}\right) \otimes P\left(\beta^{\prime}\right),
$$

where $\alpha^{\prime}$ has degree 1 and weight $p$, and $\beta^{\prime}$ has degree 2 and weight $p$. In particular, both the conjugate and the Hodge-de Rham spectral sequences for $B \alpha_{p}$ fail to degenerate.

Proof. We use the Hodge-de Rham spectral sequence and the calculation of the $E_{1}$-page coming from Proposition 4.10. Conjugate filtration considerations show that the abutment can have no terms in weights not divisible by $p$, so there must be a differential in weight 1 , which forces $d_{1}(\alpha)=u$ up to 
units. This forces all of the differentials because $\beta, s$ now have to be permanent cycles for weight and degree reasons.

Remark 4.13 (Nondegeneration of the conjugate spectral sequence for $B \alpha_{p}$ ). One can also calculate the de Rham cohomology of $B \alpha_{p}$ using the conjugate spectral sequence, giving a different proof of Proposition 4.12. Because $\alpha_{p}$ is defined over $\mathbf{F}_{p}$, we may assume $k=\mathbf{F}_{p}$, which allows us to suppress Frobenius twists. The conjugate spectral sequence takes the form

$$
E_{2}^{i, j}=H^{i}\left(B \alpha_{p}, \wedge^{j} L_{B \alpha_{p} / k}\right) \Rightarrow H_{\mathrm{dR}}^{i+j}\left(B \alpha_{p} / k\right) .
$$

The $E_{2}$-page is again calculated by Proposition 4.10, except that all weights are multiplied by $p$ (due to the implicit Frobenius twists).

First, note that $\alpha$ and $\beta$ are permanent cycles because there is no room for the differentials. Moreover, by weight considerations, $d_{2}(u)=0$, which makes $u$ a permanent cycle because the higher differentials have 0 target. The key claim is that $d_{2}(s)=\beta$ (up to units). Granting this claim, one immediately deduces the calculation of $H_{\mathrm{dR}}^{*}\left(B \alpha_{p} / k\right)$ given in Proposition 4.12, as well as the fact that both the Hodge-de Rham and conjugate spectral sequences do not degenerate (by counting dimensions).

To prove the claim $d_{2}(s)=\beta$ (up to units), we use that $B \alpha_{p}$ does not lift to $\mathbf{Z} / p^{2}$. This implies that the map ob ${ }_{B \alpha_{p}}: L_{B \alpha_{p} / k} \rightarrow \mathcal{O}[2]$ measuring the failure to lift to $W_{2}$ is nonzero. But, for any syntomic stack $\mathcal{X} / k$, the $d_{2}$ differential $H^{i}\left(\mathcal{X}, L_{\mathcal{X} / k}\right) \rightarrow H^{i+2}(\mathcal{X}, \mathcal{O})$ in the conjugate spectral sequence is just the map on $H^{i}$ induced by ob $\mathcal{X}$ (by [17, Theorem 3.5] extended to stacks). Thus, it is enough to show that the map $H^{0}\left(B \alpha_{p}, \mathrm{ob}_{B \alpha_{p}}\right)$ is nonzero. For this, write $L_{B \alpha_{p} / k} \simeq \mathcal{O} s \oplus \mathcal{O} u[-1]$ using the generators found in Proposition 4.10. We must show that the restriction of $\mathrm{ob}_{B \alpha_{p}}$ to the first factor $\mathcal{O}_{s}$ is nonzero. But the restriction of $\mathrm{ob}_{B \alpha_{p}}$ to the second factor $\mathcal{O} u[-1]$ is 0 by comparison with the analogous situation for the liftable stack $B \mathbf{G}_{a}$. Because $\mathrm{ob}_{B \alpha_{p}}$ was already shown to be nonzero, the claim follows.

Remark 4.14. Combining Proposition 4.12 (or Remark 4.13) with the approximation result in Theorem 1.2 gives a large supply of examples of smooth projective surfaces in characteristic $p$ where both the Hodge-de Rham and conjugate spectral sequences fail to degenerate.

Proposition 4.15. For all $p$, the de Rham-HP spectral sequence for $B \alpha_{p}$ degenerates.

Proof. The $E_{2}$-term is given by $E\left(\alpha^{\prime}\right) \otimes P\left(\beta^{\prime}\right) \otimes P\left(t^{ \pm 1}\right)$, where $\alpha^{\prime}, \beta^{\prime}$ are in Proposition 4.12 and $|t|=2$ has weight 0 . Because $t$ comes from the cohomology of the circle, it is a permanent cycle. Because $\alpha^{\prime}, \beta^{\prime}$ have weight $p$, one checks that $\alpha^{\prime}, \beta^{\prime}$ are forced to be permanent cycles for weight reasons. Thus, there is no room for differentials in the spectral sequence.

Remark 4.16. It follows that $\operatorname{HP}\left(B \alpha_{p} / k\right) \nsucc \mathrm{HH}\left(B \alpha_{p} / k\right)^{t S^{1}}$. In fact, the degenerate de Rham-HP spectral sequence shows that $H^{*}\left(\mathrm{HP}\left(B \alpha_{p} / k\right)\right)$ is uncountably dimensional in each degree. However, because $\mathrm{HH}\left(B \alpha_{p} / k\right)$ is coconnective and countably dimensional, it is easy to see that $\mathrm{HH}\left(B \alpha_{p} / k\right)^{t S^{1}}$ is countably dimensional in each degree.

Proposition 4.17. The crystalline cohomology of $B \alpha_{p}$ is given by

$$
H_{\text {crys }}^{*}\left(B \alpha_{p}\right) \simeq W(k)\left[\beta^{\prime}\right] / p \beta^{\prime},
$$

where $\left|\beta^{\prime}\right|=2$.

Proof. This follows from Proposition 4.12, provided that we can show that $H_{\text {crys }}^{2}\left(B \alpha_{p}\right)$ is simple $p$ torsion. But this is clear because the group scheme $\alpha_{p}$ is annihilated by $p$.

Let us collect everything we know. 
Theorem 4.18. For $B \alpha_{p}$, the following assertions hold true:

(a) The HKR spectral sequence does not degenerate. There is a nonzero differential $d_{p}: H^{0}\left(B \alpha_{p}, L_{B \alpha_{p}}\right) \rightarrow H^{p}\left(B \alpha_{p}, \wedge^{p} L_{B \alpha_{p}}\right)$.

(b) The Hodge-de Rham spectral sequence does not degenerate: There is a nonzero $d_{1}: H^{1}\left(B \alpha_{p}, \mathcal{O}\right) \rightarrow$ $H^{1}\left(B \alpha_{p}, L_{B \alpha_{p}}\right)$. Similarly, the conjugate spectral sequence does not degenerate: There is a nonzero differential $d_{2}: H^{0}\left(B \alpha_{p}, L_{B \alpha_{p} / k}\right) \rightarrow H^{2}\left(B \alpha_{p}, \mathcal{O}\right)$.

(c) The Tate spectral sequence for HP does not degenerate (there is already a nonzero $d_{2}$ ).

(d) The de Rham-HP spectral sequence degenerates.

(e) The crystalline-TP spectral sequence degenerates.

Proof.

(a) This was shown in the course of proving Proposition 4.11.

(b) This was shown in Proposition 4.12 and Remark 4.13.

(c) This was shown in Proposition 4.11.

(d) This was shown in Proposition 4.15.

(e) All terms on the $E_{2}$-page live in even degrees (Proposition 4.17), so there are no differentials.

\section{A weak Lefschetz property}

Our main goal in this section is to verify a version of the weak Lefschetz theorem for the Hodge cohomology of complete intersections in projective space (in arbitrary characteristic). In the case of a smooth complete intersection, these results are special cases of those in [18, Exposé XI.1.3]. However, in the next section, it will be crucial to have the result for singular complete intersections.

For simplicity, we work everywhere over a base field $k$. Unless specified otherwise, cotangent complexes and de Rham cohomology are computed relative to $k$.

Definition 5.1 (Hodge $d$-equivalences). We say that a map of syntomic algebraic stacks $\mathcal{X} \rightarrow \mathcal{Y}$ is a Hodge $d$-equivalence if, for each $s \geqslant 0$, we have

$$
\operatorname{cofib}\left(R \Gamma\left(\mathcal{Y}, \wedge^{s} L_{\mathcal{Y}}\right) \rightarrow R \Gamma\left(\mathcal{X}, \wedge^{s} L_{\mathcal{X}}\right)\right) \in D(k)^{\geqslant d-s} .
$$

Remark 5.2 (Consequences in de Rham and crystalline cohomology). Say $\mathcal{X} \rightarrow \mathcal{Y}$ is a Hodge $d$ equivalence of syntomic stacks over a perfect field $k$ of characteristic $p$. Then the map $R \Gamma_{\mathrm{dR}}(\mathcal{X}) \rightarrow$ $R \Gamma_{\mathrm{dR}}(\mathcal{Y})$ preserves the conjugate filtration and each graded piece has cofibre in $D^{\geqslant d}(k)$ by our assumption. It follows that the map $R \Gamma_{\mathrm{dR}}(\mathcal{X}) \rightarrow R \Gamma_{\mathrm{dR}}(\mathcal{Y})$ itself has cofibre in $D^{\geqslant d}(k)$. Passing to crystalline cohomology, this implies that the cofibre $C$ of the map $R \Gamma_{\text {crys }}(\mathcal{Y}) \rightarrow R \Gamma_{\text {crys }}(\mathcal{X})$ is in $D(W(k))^{\geqslant d}$ and, moreover, $H^{d}(C)$ is $p$-torsion free.

The main result of this section is the following result.

Proposition 5.3. Let $X$ be a d-dimensional complete intersection in $\boldsymbol{P}_{k}^{n}$. Then the inclusion map $X \rightarrow \boldsymbol{P}_{k}^{n}$ is a Hodge d-equivalence.

The argument for Proposition 5.3 is based on an induction on the codimension and, in fact, it will be convenient to prove a slightly stronger result (Corollary 5.8), based on the following two notions (which we will need only for schemes).

Definition 5.4 (KAN $d$-equivalences). Let $f: Y \rightarrow X$ be a map of syntomic $k$-schemes and let $\mathcal{L}$ be a line bundle on $X$. We say that $f$ is a Kodaira-Nakano-Akizuki $d$-equivalence (or KAN $d$-equivalence) if for each $s \geqslant 0$ and $r \geqslant 0$,

$$
\operatorname{cofib}\left(R \Gamma\left(X, \wedge^{s} L_{X} \otimes \mathcal{L}^{-r}\right) \rightarrow R \Gamma\left(Y, \wedge^{s} L_{Y} \otimes \mathcal{L}^{-r}\right)\right) \in D(k)^{\geqslant d-s} .
$$

Taking $r=0$, we see that a KAN $d$-equivalence is in particular a Hodge $d$-equivalence. 
Definition 5.5. A Kodaira pair is an $n$-dimensional $k$-scheme $Y$ and an ample line bundle $\mathcal{L}$ such that $R \Gamma\left(Y, \wedge^{s} L_{Y} \otimes \mathcal{L}^{-r}\right) \in D(k)^{\geqslant n-s}$ for all $s \geqslant 0$ and all $r>0$.

\section{Example 5.6.}

(1) Projective space $\mathbf{P}^{n}$ with any ample line bundle $\mathcal{O}_{\mathbf{P}^{n}}(h)$ (so that $h>0$ ) is a Kodaira pair.

(2) In characteristic 0 , any smooth projective variety $Y$ with an ample line bundle $L$ is a Kodaira pair, by the Kodaira-Nakano-Akizuki vanishing theorem [17, Corollary 2.11].

Proposition 5.7 (Weak Lefschetz). Let $(X, \mathcal{L})$ be a Kodaira pair of dimension d. If $i: H \rightarrow X$ is the inclusion of an effective Cartier divisor defined by a section of a positive power of $\mathcal{L}$, then

(i) the pair $\left(H, i^{*} \mathcal{L}\right)$ is a Kodaira pair and

(ii) the inclusion $i: H \rightarrow X$ is a KAN $(d-1)$-equivalence.

Proof. In the following, we write $\mathcal{O}(r)=\mathcal{L}^{r}$ for simplicity. For each $i \geqslant 0$, we consider the statements $S_{i}$ :

(a) $R \Gamma\left(H, \wedge^{i} L_{H}(-r)\right) \in D^{\geqslant d-i-1}(k)$ for $r>0$ and

(b) the map $R \Gamma\left(X, \wedge^{i} L_{X}(-r)\right) \rightarrow R \Gamma\left(H, \wedge^{i} L_{H}(-r)\right)$ has cofibre in $D^{\geqslant d-i-1}(k)$ for $r \geqslant 0$.

For all $i \geqslant 0$, the statements $S_{i}$ imply the result. We will prove $S_{i}$ by induction on $i$. Note that in the statement $S_{i}$, part (a) is actually a consequence of part (b) because $(X, \mathcal{L})$ is a Kodaira pair; however, it will be convenient to have part (a) marked separately.

For $i=0$, we use the cofibre sequence $\mathcal{O}_{X}(-H-r) \rightarrow \mathcal{O}_{X}(-r) \rightarrow i_{*} \mathcal{O}_{H}(-r)$ for any $r \geqslant 0$; we get

$$
\operatorname{cofib}\left(R \Gamma\left(X, \mathcal{O}_{X}(-r)\right) \rightarrow R \Gamma\left(H, \mathcal{O}_{H}(-r)\right)\right) \simeq R \Gamma\left(X, \mathcal{O}_{X}(-r-H)\right)[1] \in D^{\geqslant d-1}(k)
$$

by our assumption that $(X, \mathcal{L})$ is a Kodaira pair and that $\mathcal{O}(-H)=\mathcal{L}^{-t}$ for some $t>0$. This implies $S_{0}$.

For $i>0$, we first consider the factorisation of the map in question (for any $r \geqslant 0$ ),

$$
R \Gamma\left(X, \wedge^{i} L_{X}(-r)\right) \stackrel{f_{i, r}}{\longrightarrow} R \Gamma\left(H, i^{*} \wedge^{i} L_{X}(-r)\right) \stackrel{g_{i, r}}{\longrightarrow} R \Gamma\left(H, \wedge^{i} L_{H}(-r)\right) .
$$

It suffices to see that each of these maps has cofibre in $D^{\geqslant d-i-1}(k)$. The first map $f_{i, r}$ has cofibre in $D^{\geqslant d-i-1}(k)$ via the cofibre sequence

$$
R \Gamma\left(X, \wedge^{i} L_{X}(-r-H)\right) \rightarrow R \Gamma\left(X, \wedge^{i} L_{X}(-r)\right) \stackrel{f_{i, r}}{\longrightarrow} R \Gamma\left(H, i^{*} \wedge^{i} L_{X}(-r)\right)
$$

and our assumption that $(X, \mathcal{L})$ is a Kodaira pair. For the second map $g_{i, r}$, we use the conormal sequence $\mathcal{O}_{H}(-H) \rightarrow i^{*} L_{Y} \rightarrow L_{H}$ in $D(H)$ to regard $i^{*} L_{Y}$ as a (two-term) filtered object in $D(H)$. We can take exterior powers to obtain a filtration on $\wedge^{i} i^{*} L_{Y}$ (cf. the proof of [28, Prop. 25.2.4.1]); because $\mathcal{O}_{H}(-H)$ has rank 1 , this filtration degenerates to a cofibre sequence $\wedge^{i-1} L_{H}(-H) \rightarrow i^{*} \wedge^{i} L_{X} \rightarrow \wedge^{i} L_{H}$. Twisting by $-r$ and taking global sections, we obtain a cofibre sequence

$$
R \Gamma\left(H, i^{*} \wedge^{i} L_{X}(-r)\right) \stackrel{g_{i, r}}{\longrightarrow} R \Gamma\left(H, \wedge^{i} L_{H}(-r)\right) \rightarrow R \Gamma\left(H, \wedge^{i-1} L_{H}(-H-r)\right)[1] .
$$

Now part (a) of statement $S_{i-1}$ implies that the cofibre of $g_{i, r}$ belongs to $D^{\geqslant d-i-1}(k)$, as desired. This completes the proof of the statement $S_{i}$ and thus of the result.

Corollary 5.8. Let $(X, \mathcal{L})$ be a Kodaira pair. Let $i: Y \hookrightarrow X$ be a d-dimensional complete intersection of sections of powers of $\mathcal{L}$ (in particular, those sections form a regular sequence). Then $\left(Y, i^{*} \mathcal{L}\right)$ is a Kodaira pair and $i: Y \rightarrow X$ is a KAN d-equivalence (in particular, a Hodge d-equivalence).

Proof. Observe that the composite of a KAN-m-equivalence and a KAN-n-equivalence is a KAN$\min (m, n)$-equivalence. Therefore, the result follows by iteratively applying the weak Lefschetz (Proposition 5.7). 
Example 5.9 (Smooth complete intersections). Let $X$ be a smooth complete intersection of dimension $d$ inside $\mathbf{P}^{n}$ and let $i: H \hookrightarrow X$ be a smooth hypersurface. According to Corollary 5.8, $X, H$ are Kodaira pairs with respect to the line bundle $\mathcal{O}(1)$, and $i$ is a $\mathrm{KAN}(d-1)$-equivalence. In fact, this is well known: In characteristic 0, this follows from the Kodaira-Akizuki-Nakano vanishing theorem [17, Corollary 2.11]. In positive characteristic, one can use Deligne's computations to obtain the same result; see [18, Exposé XI.1.3].

In the remainder of this section, we record two more basic properties of Hodge cohomology and Hodge $d$-equivalences; these will be used essentially in the next section.

Proposition 5.10 (Preservation of Hodge $d$-equivalences). Suppose that $X \rightarrow Y$ is a Hodge $d$ equivalence of syntomic $k$-schemes.

(1) For any syntomic $k$-scheme $Z, X \times_{k} Z \rightarrow Y \times_{k} Z$ is a Hodge d-equivalence.

(2) If $G$ is an affine $k$-group scheme of finite type (and thus $G$ is syntomic) that acts on both $X$ and $Y$ equivariantly for the map $X \rightarrow Y$, then the map $[X / G] \rightarrow[Y / G]$ of quotient stacks is a Hodge d-equivalence.

Proof. Part (1) follows from the Künneth formula in Hodge cohomology; we have

$$
R \Gamma\left(X \times_{k} Z, \wedge^{s} L_{X \times_{k} Y}\right) \simeq \bigoplus_{a+b=s} R \Gamma\left(X, \wedge^{a} L_{X}\right) \otimes_{k} R \Gamma\left(Z, \wedge^{b} L_{Z}\right)
$$

and similarly for $Y \times_{k} Z$. Note now that $R \Gamma\left(Z, \wedge^{b} L_{Z}\right)$ belongs to $D(k)^{\geqslant-b}$ because $Z$ is syntomic. Therefore, the map $R \Gamma\left(X, \wedge^{a} L_{X}\right) \otimes_{k} R \Gamma\left(Z, \wedge^{b} L_{Z}\right) \rightarrow R \Gamma\left(Y, \wedge^{a} L_{Y}\right) \otimes_{k} R \Gamma\left(Z, \wedge^{b} L_{Z}\right)$ has cofibre in $D(k)^{\geqslant d-a-b}=D(k)^{\geqslant d-s}$, as desired.

For part (2), consider the resolution $\cdots \underset{\rightarrow}{\rightarrow} G \times X \rightrightarrows X$ of the stack $[X / G]$ and similarly for $[Y / G]$. By hypothesis and Proposition 5.10, the induced map of cosimplicial objects $R \Gamma\left(G^{\bullet} \times{ }_{k} Y, \wedge^{s} L_{G^{\bullet} \times{ }_{k} Y}\right) \rightarrow$ $R \Gamma\left(G^{\bullet} \times X, \wedge^{s} L_{G^{\bullet} \times{ }_{k} X}\right)$ has levelwise cofibre in $D(k)^{\geqslant d-s}$. Because $D(k)^{\geqslant d-s}$ is closed under limits in $D(k)$, the result now follows by taking the limit.

Proposition 5.11 (Projective bundle formula). Let $\mathcal{X}$ be a syntomic stack. Given an $n$-dimensional vector bundle $V$ over $\mathcal{X}$, let $\mathcal{Y}$ be the associated projective bundle over $\mathcal{X}$. Then there exists a class $c_{1} \in H^{1}\left(\mathcal{Y}, L_{\mathcal{Y}}\right)$ such that $H^{*}\left(\mathcal{Y}, \wedge^{*} L_{\mathcal{Y}}\right)$ is a free module over $H^{*}\left(\mathcal{X}, \wedge^{*} L_{\mathcal{X}}\right)$ on $1, c_{1}, \ldots, c_{1}^{n-1}$.

Proof. The class $c_{1}$ is the first Chern class (in Hodge cohomology) of the tautological line bundle $\mathcal{O}(1)$ on $\mathcal{Y}$, which is defined via pullback from the induced map $\mathcal{Y} \rightarrow B \mathbf{G}_{m}$ classifying $\mathcal{O}(1)$. The result then asserts that for each $i$, the map

$$
\bigoplus_{j=0}^{n-1} R \Gamma\left(\mathcal{X}, \wedge^{i-j} L_{\mathcal{X}}\right)[-j] \rightarrow R \Gamma\left(\mathcal{Y}, \wedge^{i} L_{\mathcal{Y}}\right)
$$

obtained as multiplication by $c_{1}^{j}$ on the $j$ th factor, is an equivalence. This assertion is local on $\mathcal{X}$, whence we reduce to the case of $\mathcal{X}$ an affine scheme and $V$ a trivial bundle, for which the result is classical.

\section{Approximation of classifying spaces and failure of HKR}

Using our study of Hodge $d$-equivalences from the previous section, we prove Theorem 1.2 from the Introduction. The ideas here are not new and go back to work of Serre [36] and Totaro [42]; we also use arguments from [11].

Proof of Theorem 1.2. First, we assume that $G$ is a finite group scheme. We claim that there is a finitedimensional representation $V$ of $G$ and a $d$-dimensional complete intersection $Z \subseteq \mathbf{P}(V)$ such that $Z$ is stable under the $G$-action, $G$ acts freely on $Z$ and $Z / G \simeq[Z / G]$ is smooth and projective. This is a 
standard argument involving an application of a Bertini-type theorem for the quotient variety $\mathbf{P}(V) / G$ (see, e.g., [11, 2.7-2.9]). ${ }^{4}$ Having found $Z$, we note that $Z \hookrightarrow \mathbf{P}(V)$ is a Hodge $d$-equivalence by Proposition 5.3. Therefore, the induced map $Z / G \simeq[Z / G] \hookrightarrow[\mathbf{P}(V) / G]$ on quotient stacks is a Hodge $d$-equivalence by Proposition 5.10. The theorem now follows from Proposition 5.11 by taking $X=Z / G$.

Now suppose that $G$ is geometrically reductive. For each $r$, we let $G_{r} \subset G$ be the kernel of the $r$ th Frobenius on $G$. According to [24, Cor. II.4.12], for any finite-dimensional $G$-representation $V$, we have that the cohomology groups $H^{i}(G, V), H^{i}\left(G_{r}, V\right)$ are finite-dimensional for $i \geqslant 0$, and $H^{i}(G, V) \simeq \lim _{r} H^{i}\left(G_{r}, V\right)$. We claim that for all $i, j, r \geqslant 0$, the vector spaces $H^{i}\left(B G, \wedge^{j} L_{B G}\right)$ and $H^{i}\left(B G_{r}, \wedge^{j}, \overleftarrow{L}_{B G_{r}}\right)$ are finite-dimensional and

$$
H^{i}\left(B G, \wedge^{j} L_{B G}\right) \simeq \lim _{\longleftarrow} H^{i}\left(B G_{r}, \wedge^{j} L_{B G_{r}}\right)
$$

By finite-dimensionality, this implies that for any $i, j$, the map $H^{i}\left(B G, \wedge^{j} L_{B G}\right) \rightarrow H^{i}\left(B G_{r}, \wedge^{j} L_{B G_{r}}\right)$ is injective for $r \gg 0$. From this, we reduce the case of reductive $G$ to finite $G$ treated above.

To prove the claim (4), we observe that by functoriality we have maps (where each object belongs to the appropriate derived category, and the maps are compatible in the natural sense)

$$
\operatorname{co-Lie}(G) \rightarrow \cdots \rightarrow \operatorname{co-Lie}\left(G_{r+1}\right) \rightarrow \operatorname{co}-\operatorname{Lie}\left(G_{r}\right) \rightarrow \cdots \rightarrow \operatorname{co}-\operatorname{Lie}\left(G_{1}\right) .
$$

By the description of the $G_{r}$ as Frobenius kernels, we find that each of these maps is an isomorphism on $H^{0}$ and induces the zero map on $H^{-1}$. Taking rational cohomology, we find easily that

$$
\underset{\lim }{\longleftarrow} R \Gamma\left(G_{r}, \operatorname{Sym}^{i} \operatorname{co}-\operatorname{Lie}\left(G_{r}\right)\right) \simeq \lim _{\longleftarrow}^{\lim } R \Gamma\left(G_{r}, H^{0}\left(\operatorname{Sym}^{i} \operatorname{co-Lie}\left(G_{r}\right)\right)\right)=\lim ^{\lim } R \Gamma\left(G_{r}, \operatorname{Sym}^{i} \operatorname{co}-\operatorname{Lie}(G)\right)
$$

and all cohomologies are finite-dimensional in each degree. Combining with [24, Cor. II.4.12] and the decomposition (3), we conclude $R \Gamma\left(B G, \wedge^{i} L_{B G}\right) \simeq \lim _{r} R \Gamma\left(B G, \wedge^{i} L_{B G_{r}}\right)$. This yields (4), because finite-dimensionality prevents the existence of nonzero $\lim ^{1}$ terms.

Remark 6.1. The proof of Theorem 1.2 given above proves a slightly stronger statement in the case of finite group schemes $G$ : we find approximations $X \rightarrow B G$ as in Theorem 1.2 such that $H^{*}\left(X, \wedge^{*^{\prime}} L_{X}\right)$ is free as a bigraded $H^{*}\left(B G, \wedge^{*^{\prime}} L_{B G}\right)$-module in total degrees $*+*^{\prime} \leqslant d$ on classes $c^{i} \in H^{i}\left(X, \wedge^{i} L_{X}\right)$ for $0 \leqslant i \leqslant\left\lfloor\frac{d}{2}\right\rfloor$.

We can now prove that several spectral sequences as explained earlier are nondegenerate.

Proof of Theorem 1.1. Choose $G=\alpha_{p}$ or $G=\mu_{p}$. In each case, we find that there is a nonzero differential $d_{p}: H^{0}\left(B G, \wedge^{1} L_{B G}\right) \rightarrow H^{p}\left(B G, \wedge^{p} L_{B G}\right)$ in the HKR spectral sequence. If we choose $X \rightarrow B G$ as in Theorem 1.2 to have dimension $2 p$, then we find that the differential $d_{p}: H^{0}\left(X, \Omega_{X}^{1}\right) \rightarrow$ $H^{p}\left(X, \Omega_{X}^{p}\right)$ is nonzero, as desired.

Theorem 6.2. Let $k$ be a perfect field of characteristic $p>0$.

(a) There exists a smooth projective $2 p$-fold such that the de Rham-HP spectral sequence does not degenerate.

(b) There exists a smooth projective variety such that the crystalline-TP spectral sequence does not degenerate.

(c) There exists a smooth projective variety $X$ such that the filtration on $H^{*}(T P(X))$ arising from the crystalline-TP spectral sequence is not split.

Proof. For (a), let $X \rightarrow B \mu_{p}$ be a smooth projective approximation as in Theorem 1.2 for $d=2 p$, so $H^{t}\left(B \mu_{p}, \wedge^{s} L_{B \mu_{p}}\right) \rightarrow H^{t}\left(X, \wedge^{s} L_{X}\right)$ is injective for $s+t \leqslant 2 p$. By the results of Subsection 4.1, we see that in the de Rham-HP spectral sequence for $B \mu_{p}$, there is a nonzero differential $d_{p}: H_{\mathrm{dR}}^{1}\left(B \mu_{p}\right) \rightarrow$

${ }^{4}$ The argument relies on Bertini-type theorems; in case $k$ is finite, one can use Bertini theorems in the form of [31]. 
$H_{\mathrm{dR}}^{2 p}\left(B \mu_{p}\right)$. Thus, by naturality, there is also a nonzero differential in the de Rham-HP spectral sequence for $X$, as desired.

Now, for part (b), by Lemma 4.8, the crystalline-TP spectral sequence for $B\left(\mu_{p} \times \mu_{p}\right)$ does not degenerate. In particular, from the proof we see that there is some first nonzero differential $d_{r}: H_{\text {crys }}^{1}\left(B\left(\mu_{p} \times \mu_{p}\right)\right) \rightarrow H_{\text {crys }}^{2 r}\left(B\left(\mu_{p} \times \mu_{p}\right)\right)$. Choose a smooth projective approximation $X \rightarrow B\left(\mu_{p} \times \mu_{p}\right)$ with $d=2 r$. Then, the crystalline-TP spectral sequence does not degenerate for $X$.

To prove part (c), we take a smooth projective approximation to $B \mu_{p}$ as in 1.2 with $d>2 p$. Then, $H_{\text {crys }}^{t}\left(B \mu_{p}\right) \rightarrow H_{\text {crys }}^{t}(X)$ is injective for $t \leqslant 2 p$. In particular, in this range, the images of the classes in $H_{\text {crys }}^{t}\left(B \mu_{p}\right)$ in $H_{\text {crys }}^{t}(X)$ are permanent cycles. Because the extensions in the spectral sequence are nontrivial for $B \mu_{p}$, they also must be nontrivial for $X$.

Remark 6.3 (Obtaining liftable examples). Take $G=\mu_{p}$ in the proof of Theorem 1.1 given above. Because the group scheme $\mu_{p}$ admits a lift (even a unique one) to $W_{2}(k)$, one can show that the smooth projective variety $X$ used in the proof of Theorem 1.1 (coming from Theorem 1.2) also lifts to $W_{2}(k)$, thus yielding a liftable example where the HKR spectral sequence does not degenerate. A similar remark applies to Theorem 6.2.

\section{Hochschild homology and the dlog map}

The construction of Hochschild homology and the HKR spectral sequence allows for a twisted version, via an Azumaya algebra or a class in the Brauer group. In this section, we describe this spectral sequence.

Throughout, we fix a base commutative ring $k$. The starting point is the following result from [14], stating that Hochschild homology groups cannot distinguish between Azumaya algebras over affine schemes.

Theorem 7.1 (Cortiñas-Weibel [14]). Suppose that $R$ is a k-algebra and $\mathcal{A}$ is an Azumaya $R$ algebra. Then there is a functorial (in $R, \mathcal{A})$ isomorphism of $H^{*}(H H(R / k))$-modules, $H^{*}(H H(\mathcal{A} / k)) \simeq$ $H^{*}(H H(R / k))$.

Corollary 7.2 (The twisted HKR theorem). Let $R$ be a smooth $k$-algebra and let $\mathcal{A}$ be an Azumaya $R$-algebra. Then there is a natural isomorphism $H^{*}(H H(\mathcal{A} / k)) \simeq \Omega_{R / k}^{-*}$.

The isomorphism $H^{*}(\mathrm{HH}(\mathcal{A} / k)) \simeq H^{*}(\mathrm{HH}(R / k))$ appearing in Corollary 7.2 can be chosen functorially at the level of cohomology groups but not at the level of complexes. Globalising, this leads to the following constructions.

Construction 7.3 (Twisted Hochschild homology). Let $X$ be a $k$-scheme and let $\mathcal{A}$ be an Azumaya algebra over $X$ (i.e., a sheaf of Azumaya $\mathcal{O}_{X}$-algebras). For each étale map from an affine, $\operatorname{Spec}(R) \rightarrow X$, we obtain an Azumaya $R$-algebra $\mathcal{A}_{R}$ and can form the Hochschild homology $\operatorname{HH}\left(\mathcal{A}_{R} / k\right)$. As $R$ varies, we obtain an object of $D(X)$, denoted $\mathbf{H H}(-/ k)_{\mathcal{A}}$. We write $\operatorname{HH}(\mathcal{A} / k)=R \Gamma\left(X, \mathbf{H H}(-/ k)_{\mathcal{A}}\right)$ for the global sections of $\mathbf{H H}(-/ k)_{\mathcal{A}}$. We call this construction the $\mathcal{A}$-twisted Hochschild homology of $X$.

Construction 7.4 (The twisted HKR spectral sequence). Let $\mathcal{A}$ be an Azumaya algebra over the smooth $k$-scheme $X$. For each étale map $\operatorname{Spec}(R) \rightarrow X$, we have functorial isomorphisms of Hochschild homology groups $H^{*}\left(\mathrm{HH}\left(\mathcal{A}_{R} / k\right)\right) \simeq \Omega_{R / k}^{*}$ by Corollary 7.2. Globalising, we obtain a spectral sequence

$$
E_{2}^{s, t}=H^{s}\left(X, \Omega_{X / k}^{-t}\right) \Rightarrow H^{s+t}(\mathrm{HH}(\mathcal{A} / k)),
$$

the $\mathcal{A}$-twisted HKR spectral sequence.

The main result of this section (Proposition 7.7) is an identification of the first differential in this spectral sequence. In proving the result, we will also clarify the precise choice of isomorphism in Theorem 7.1. 
Construction 7.5 (Étale twisted $K$-theory). Let $\mathcal{A}$ be an Azumaya algebra over the $k$-scheme $X$, representing a Brauer class $\alpha$. We consider the $\mathcal{A}$-twisted étale $K$-theory $\mathbf{K}^{\text {ét }}(-)_{\mathcal{A}}$, defined as the étale sheafification over $X$ of $R \mapsto \mathrm{K}\left(\mathcal{A}_{R}\right)$, where $\mathrm{K}\left(\mathcal{A}_{R}\right)$ denotes the algebraic K-theory spectrum of $\mathcal{A}_{R}$. Because Hochschild homology has étale descent [44, 14], the object $\mathbf{K}^{\text {et }}(-)_{\mathcal{A}}$ is equipped with a trace map (obtained by sheafifying the Dennis trace)

$$
\mathbf{K}^{\text {ét }}(-)_{\mathcal{A}} \rightarrow \mathbf{H H}(-/ k)_{\mathcal{A}} .
$$

Construction 7.6 (The normalisation of the isomorphism $\mathrm{HH}_{*}(R / k) \simeq \mathrm{HH}_{*}(\mathcal{A} / k)$ ). Let $\mathcal{A}$ be an Azumaya algebra over the $k$-scheme $X$. Here we construct explicitly the isomorphism of Theorem 7.1.

According to the étale descent theorem [44, 14], it follows that $H^{*}\left(\mathbf{H H}(-/ k)_{\mathcal{A}}\right)$ define quasicoherent sheaves on the étale site of $X$, which consequently have no higher cohomology. According to [2, Sec. 5], the étale sheafified homotopy groups $\pi_{i} \mathbf{K}^{\text {ét }}(-)_{\mathcal{A}}$ are canonically isomorphic to the untwisted sheafified homotopy groups $\pi_{i} \mathbf{K}(-)$. In particular, we have a canonical isomorphism $\pi_{0} \mathbf{K}^{\text {ét }}(-)_{\mathcal{A}} \cong \mathbf{Z}$. The trace map thus gives a map $\mathbf{Z} \rightarrow \pi_{0}\left(\mathbf{H H}(-/ k)_{\mathcal{A}}\right)$ ), which one checks étale locally (over which $\mathcal{A}$ is trivial) to be a generator; that is, to induce an isomorphism $H^{*}\left((\mathbf{H H}(-/ k)) \cong H^{*}\left(\mathbf{H H}(-/ k)_{\mathcal{A}}\right)\right.$.

Now we identify the first differential in the $\mathcal{A}$-twisted HKR spectral sequence. For this, we use the map dlog: $\mathbf{G}_{m} \rightarrow \Omega_{(-) / k}^{1}$ of étale sheaves. In particular, for any scheme $X$, it defines a map $H^{i}\left(X, \mathbf{G}_{m}\right) \rightarrow H^{i}\left(X, \Omega_{X / k}^{1}\right)$. This map arises explicitly in the Dennis trace map. Namely, if $R$ is a smooth $k$-algebra, then the map

$$
R^{\times} \rightarrow K_{1}(R) \rightarrow H^{-1}(\mathrm{HH}(R / k)) \simeq \Omega_{R / k}^{1}
$$

is given by dlog, in view of [35, Theorem 6.2.16].

Proposition 7.7 (The first differential in twisted HKR). Let $\mathcal{A}$ be an Azumaya algebra on a $k$-scheme $X$ with class $\alpha \in H^{2}\left(X, \boldsymbol{G}_{m}\right)$. Then, the differential

$$
d_{2}^{\alpha}: H^{0}\left(X, \mathcal{O}_{X}\right) \rightarrow H^{2}\left(X, \Omega_{X / k}^{1}\right)
$$

in the $\mathcal{A}$-twisted HKR spectral sequence for $H H(\mathcal{A} / k)$ sends 1 to $\operatorname{dlog} \alpha$.

Proof. We have a natural trace map of étale sheaves $\mathbf{K}^{\text {ét }}(-)_{\mathcal{A}} \rightarrow \mathbf{H H}(-/ k)_{\mathcal{A}}$ and hence an induced map of étale descent spectral sequences. The argument of [2, Proposition 5.1] provides isomorphisms $\pi_{i} \mathbf{K}^{\text {ét }}(-)_{\mathcal{A}} \cong \pi_{i} \mathbf{K}^{\text {ét }}(-)$ and Corollary 7.2 provides isomorphisms $H^{i}\left(\mathbf{H H}(-/ k)_{\mathcal{A}}\right) \cong H^{i}(\mathbf{H H}(-/ k)) ;$ by construction, these are compatible with the $\alpha$-twisted and untwisted trace. Thus, $\pi_{0} \mathbf{K}^{\text {ét }}(-)_{\mathcal{A}} \cong \mathbf{Z}$, $\pi_{1} \mathbf{K}^{\text {ét }}(-)_{\mathcal{A}} \cong \mathbf{G}_{m}, H^{0}\left(\mathbf{H H}(-/ k)_{\mathcal{A}}\right) \cong \mathcal{O}_{X}$ and $H^{-1}\left(\mathbf{H H}(-/ k)_{\mathcal{A}}\right) \cong \Omega_{X}^{1}$. Because the trace induces dlog in degree 1 , we have a commutative square

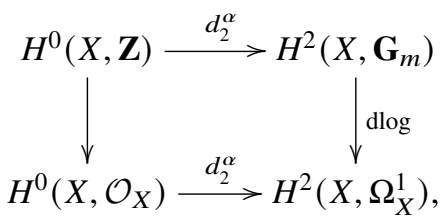

where $d_{2}^{\alpha}$ denotes the differential in the spectral sequences converging to the $\alpha$-twisted forms of étale $K$-theory and Hochschild homology. By the main result of [1], we have $d_{2}^{\alpha}(1)=\alpha$ for the top horizontal arrow. Because the left vertical arrow sends 1 to 1 , the corollary follows.

Remark 7.8. Let $k$ be a perfect field of characteristic $p>0$. If $X$ is a smooth proper $k$-scheme and $\mathcal{A}$ is an Azumaya algebra on $X$ with Brauer class $\alpha \in H^{2}\left(X, \mathbf{G}_{m}\right)$ such that $\operatorname{dlog} \alpha \neq 0$ in $H^{2}\left(X, \Omega_{X}^{1}\right)$, then the $\mathcal{A}$-twisted HKR spectral sequence does not degenerate, contrary to what happens in the untwisted case when $\operatorname{dim} X \leqslant p$ (see [7]). In the next two sections, we will find examples. 
Remark 7.9 (Cohomological Brauer classes). The $\mathcal{A}$-twisted Hochschild homology and HKR spectral sequence only depend on the Brauer class $\alpha \in H^{2}\left(X, \mathbf{G}_{m}\right)$, and one can define twisted Hochschild homology purely in terms of $\alpha$ (even when it is not representable by an Azumaya algebra). Indeed, one can construct twisted Hochschild homology as the Hochschild homology of representing derived Azumaya algebras [39, 5]. Moreover, twisted Hochschild homology and the associated twisted HKR spectral sequence (along the lines of Definition 3.1(a)) exist for any class $\alpha \in H^{2}\left(\mathcal{X}, \mathbf{G}_{m}\right)$ for any syntomic $k$-stack $\mathcal{X}$.

\section{8. $\mathrm{PGL}_{n}$}

We will give two different approaches to constructing counterexamples to the degeneration of the twisted HKR spectral sequence as in Theorem 1.3. The first, and most naive, is to take suitable approximations to $B \mathbf{P G L} \mathbf{L}_{p}$. This method produces smooth projective 3-folds. Second, for $p=2$, we note that classical Enriques surfaces give examples. In this section, we describe the first approach.

For the following argument, it will be convenient to use (very mildly) the language of higher stacks: In particular, we will want to consider $B^{2} \mathbf{G}_{m}$ as a higher stack and regard Hodge cohomology as sheaf cohomology. We briefly review this language below (in the form of sheaves of spaces). For simplicity, we will restrict to smooth schemes, because this is all we will need henceforth.

Definition 8.1 (Sheaves of spaces or higher stacks). Let $k$ be a perfect field. Consider the category $\mathrm{Sm}_{k}^{\text {aff }}$ of affine smooth $k$-schemes, equipped with the étale topology. We can consider the $\infty$-category $\operatorname{Shv}\left(\mathrm{Sm}_{k}^{\text {aff }}\right)$ of sheaves of spaces on $\mathrm{Sm}_{k}^{\text {aff }}$; see [27] for a detailed treatment of sheaves of spaces. Because $\operatorname{Shv}\left(\operatorname{Sm}_{k}^{\text {aff }}\right)$ is an $\infty$-category, there is a well-defined homotopy type $\operatorname{Map}_{\operatorname{Shv}\left(\operatorname{Sm}_{k}^{\text {aff }}\right)}(X, Y)$ for any two objects $X, Y \in \operatorname{Shv}\left(\operatorname{Sm}_{k}^{\text {aff }}\right)$.

Example 8.2 (Examples of higher stacks).

(a) Any smooth algebraic stack $\mathcal{X}$ over $k$ yields (and is determined by) an object $\operatorname{Shv}\left(\operatorname{Sm}_{k}^{\text {aff }}\right)$ via the groupoid-valued functor of points.

(b) For any smooth commutative group scheme $G$ over $k$ and any $n \geqslant 0$, we obtain a higher stack $K(G, n) \in \operatorname{Shv}\left(\operatorname{Sm}_{k}^{\text {aff }}\right)$. Explicitly, $K(G, n)$ is the étale sheafification of the functor that sends a smooth $k$-algebra $R$ to the Eilenberg-MacLane space $K(G(R), n)$.

(c) Fix $i \geqslant 0$. Consider the functor $\Omega^{i}$ that sends a smooth $k$-algebra $R$ to the differential forms $\Omega_{R}^{i}$. For $n \geqslant 0$, we obtain a sheaf of spaces on $\operatorname{Sm}_{k}^{\text {aff }}, K\left(\Omega^{i}, n\right) \in \operatorname{Shv}\left(\operatorname{Sm}_{k}^{\text {aff }}\right)$, such that $K\left(\Omega^{i}, n\right)(R)$ is the Eilenberg-MacLane space $K\left(\Omega_{R}^{i}, n\right)$; here we do not need to étale sheafify further.

The language of higher stacks will be useful for us because one has a good internal theory of cohomology.

Construction 8.3 (Higher group cohomology). Let $G$ be a smooth commutative $k$-group scheme. Given an object $T \in \operatorname{Shv}\left(\mathrm{Sm}_{k}^{\text {aff }}\right)$, we have the cohomology groups (for $n \geqslant 0$ )

$$
H^{n}(T, G)=\pi_{0} \operatorname{Map}_{\operatorname{Shv}\left(\operatorname{Sm}_{k}^{\mathrm{aff}}\right)}(T, K(G, n))
$$

this agrees with the usual definition (étale cohomology) in case $T$ is representable by a smooth $k$-scheme (cf. also [28, Cor. 2.1.2.3]). By delooping, we can regard these as the cohomology groups of a complex $R \Gamma(T, G)$.

Construction 8.4 (Hodge cohomology as sheaf cohomology). Let $\mathcal{X}$ be a smooth algebraic stack over $k$, which we regard as an object of $\operatorname{Shv}\left(\operatorname{Sm}_{k}^{\text {aff }}\right)$ as above. Then we have natural isomorphisms

$$
H^{n}\left(\mathcal{X}, \wedge^{i} L_{\mathcal{X}}\right)=\pi_{0} \operatorname{Map}_{\operatorname{Shv}\left(\operatorname{Sm}_{k}^{\mathrm{aff}}\right)}\left(\mathcal{X}, K\left(\Omega^{i}, n\right)\right)
$$


Example 8.5 (The degree 2 class on $\left.B \mathbf{P G L} \mathbf{L}_{n}\right)$. We have a natural map $B \mathbf{P G L}_{n} \rightarrow K\left(\mathbf{G}_{m}, 2\right)$. Explicitly, $B \mathbf{G}_{m}$ defines a commutative group object of $\operatorname{Shv}\left(\mathrm{Sm}_{k}^{\text {aff }}\right)$ acting centrally on $B \mathbf{G} \mathbf{L}_{n} ; B \mathbf{P G} \mathbf{L}_{n}$ is the quotient of $B \mathbf{G}_{m}$ acting on $B \mathbf{G} \mathbf{L}_{n}$. We thus obtain a fibre sequence $B \mathbf{G} \mathbf{L}_{n} \rightarrow B \mathbf{P G} \mathbf{L}_{n} \rightarrow K\left(\mathbf{G}_{m}, 2\right)$; that is, a class in $H^{2}\left(B \mathbf{P G} \mathbf{L}_{n}, \mathbf{G}_{m}\right)$.

Example 8.6 (The dlog map and its delooping). For any smooth $k$-algebra $R$, we have a homomorphism of abelian groups dlog: $\mathbf{G}_{m}(R) \rightarrow \Omega_{R}^{1}$ given by $\operatorname{d} \log (f)=\frac{d f}{f}$. Sheafifying and delooping twice yields a natural map $B^{2} \operatorname{dlog}: K\left(\mathbf{G}_{m}, 2\right) \rightarrow K\left(\Omega^{1}, 2\right)$.

Proposition 8.7. If $n$ is divisible by $p$, then the composite map BPG $\boldsymbol{L}_{n} \rightarrow K\left(\boldsymbol{G}_{m}, 2\right) \stackrel{B^{2} \text { dlog }}{\longrightarrow} K\left(\Omega^{1}, 2\right)$ is not null-homotopic in $\operatorname{Shv}\left(\operatorname{Sm}_{k}^{a f f}\right)$.

Proof. The map $B^{2}$ dlog is clearly not null-homotopic (because its double looping is nonzero). Our claim is then that the map $H^{2}\left(K\left(\mathbf{G}_{m}, 2\right), \Omega^{1}\right) \rightarrow H^{2}\left(B \mathbf{P G} \mathbf{L}_{n}, \Omega^{1}\right)=H^{2}\left(B \mathbf{P G} \mathbf{L}_{n}, L_{B \mathbf{P G}}\right)$ is injective. Indeed, the map $B \mathbf{P G} \mathbf{L}_{n} \rightarrow K\left(\mathbf{G}_{m}, 2\right)$ is obtained from the map $B \mathbf{G L}_{n} \rightarrow *$ by taking homotopy orbits by $B \mathbf{G}_{m}$. Thus, we have a commutative diagram of fibre sequences,

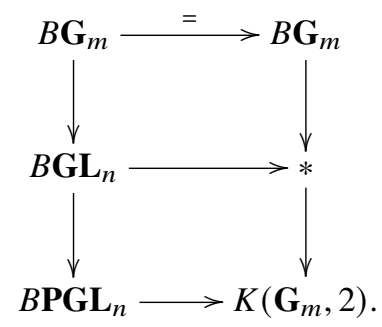

Given smooth stacks $\mathcal{X}, \mathcal{Y}$ with $R \Gamma(\mathcal{X}, \mathcal{O})=R \Gamma(\mathcal{Y}, \mathcal{O})=k$, the Künneth formula in Hodge cohomology yields $R \Gamma\left(\mathcal{X} \times \mathcal{Y}, L_{\mathcal{X} \times \mathcal{Y}}\right)=R \Gamma\left(\mathcal{X}, L_{\mathcal{X}}\right) \oplus R \Gamma\left(\mathcal{Y}, L_{\mathcal{Y}}\right)$. The diagram of fibre sequences expresses $B \mathbf{P G L} \mathbf{L}_{n}$ as the geometric realisation of a simplicial stack given as the bar construction of $B \mathbf{G}_{m}$ acting on $B \mathbf{G L}_{n}$; that is, $\cdots \underset{\rightarrow}{\rightarrow} B \mathbf{G L}_{n} \times B \mathbf{G}_{m} \rightrightarrows B \mathbf{G L}_{n}$. Similarly, $K\left(\mathbf{G}_{m}, 2\right)$ is the bar construction of $B \mathbf{G}_{m}$ acting on a point. Applying the cochains functor $R \Gamma\left(\cdot, \Omega^{1}\right)$, which carries these simplicial resolutions to totalisations, we find that these two observations imply that both vertical sequences in (7) are carried to fibre sequences. That is,

$$
\begin{gathered}
R \Gamma\left(B \mathbf{P G} \mathbf{L}_{n}, \Omega^{1}\right) \simeq \operatorname{fib}\left(R \Gamma\left(B \mathbf{G L}_{n}, \Omega^{1}\right) \rightarrow R \Gamma\left(B \mathbf{G}_{m}, \Omega^{1}\right)\right), \\
R \Gamma\left(K\left(\mathbf{G}_{m}, 2\right), \Omega^{1}\right) \simeq \operatorname{fib}\left(0 \rightarrow R \Gamma\left(B \mathbf{G}_{m}, \Omega^{1}\right)\right) .
\end{gathered}
$$

Now we have the following computation in Hodge cohomology: $R \Gamma\left(B \mathbf{G} \mathbf{L}_{n}, \Omega^{1}\right)=$ $R \Gamma\left(B \mathbf{G L}_{n}, L_{B \mathbf{G L}_{n}}\right) \simeq k[-1]$; this is a classical calculation, and a modern reference for a much more general statement is [42, Theorem 10.2]. Furthermore, the diagonal map $B \mathbf{G}_{m} \rightarrow B \mathbf{G L}_{n}$ induces the map $k[-1] \rightarrow k[-1]$ given by multiplication by $n$. This follows from the explicit expression of Hodge cohomology given in this case (i.e., as analogous to singular cohomology; cf. [42, Theorem 4.1]). We obtain that $R \Gamma\left(B \mathbf{P G L}_{n}, \Omega^{1}\right)$ is the two-term complex $k \stackrel{n}{\rightarrow} k$ in degrees 1 and 2 and $R \Gamma\left(K\left(\mathbf{G}_{m}, 2\right), \Omega^{1}\right)=k[-2]$. By naturality of the above diagrams, we obtain that the map $R \Gamma\left(K\left(\mathbf{G}_{m}, 2\right), \Omega^{1}\right) \rightarrow R \Gamma\left(B \mathbf{P G} \mathbf{L}_{n}, \Omega^{1}\right)$ is an isomorphism in $H^{2}$ : the generating classes in both sides arise by coboundary from $H^{1}\left(B \mathbf{G}_{m}, \Omega^{1}\right)$.

Now, we can prove Theorem 1.3 from the introduction.

Proof of Theorem 1.3. Take a smooth projective $X$ with $d=3$ in Theorem 1.2 for $G=\mathbf{P G L}_{p}$. According to Proposition 8.7, the composite map $B \mathbf{P G L} \mathbf{L}_{p} \rightarrow K\left(\mathbf{G}_{m}, 2\right) \rightarrow K\left(\Omega^{1}, 2\right)$ is not null-homotopic and defines a nontrivial class (indeed, the generator) of $H^{2}\left(B \mathbf{P G L} \mathbf{L}_{p}, L_{\mathbf{P G L}}\right)$. The pullback of this class under the map $X \rightarrow \mathbf{P G L}_{p}$ is nonzero by construction. Therefore, by naturality, the pullback of the 
canonical class in $H^{2}\left(X, \mathbf{G}_{m}\right)$ maps via dlog to an exact order $p$ class in $H^{2}\left(X, \Omega_{X}^{1}\right)$. We are done by Proposition 7.7: there is a nonzero $d_{2}$-differential in the twisted HKR spectral sequence.

\section{Classical Enriques surfaces in characteristic 2}

Let $k$ be an algebraically closed field of characteristic 2. For background on Enriques surfaces in positive characteristic (see [13] and [22, Section II.7.3]). A smooth proper surface $X$ over $k$ is Enriques if its canonical class, $\omega_{X}$, is algebraically equivalent to zero and if its second Betti number is $B_{2}=10$. An Enriques surface is classical if $H^{1}\left(X, \mathcal{O}_{X}\right)=0$. In this case, $H^{2}\left(X, \mathcal{O}_{X}\right)=0, \omega_{X}$ is not trivial, and $\omega_{X}^{\otimes 2} \cong \mathcal{O}_{X}$.

Recall that for smooth schemes over any perfect field of characteristic $p>0$ we have exact sequences of étale sheaves

$$
1 \rightarrow \mathbf{G}_{m} \stackrel{p^{n}}{\longrightarrow} \mathbf{G}_{m} \rightarrow v_{n}(1) \rightarrow 1
$$

for all $n$. These induce short exact sequences

$$
0 \rightarrow \operatorname{Br}(X) / p^{n} \rightarrow H^{2}\left(X, v_{n}(1)\right) \rightarrow H^{3}\left(X, \mathbf{G}_{m}\right)\left[p^{n}\right] \rightarrow 0
$$

for each $n$.

Lemma 9.1. If $X$ is a classical Enriques surface over an algebraically closed field of characteristic 2, then $\operatorname{Br}(X)=Z / 2$. In particular, the nonzero class $\alpha \in \operatorname{Br}(X)$ defines a nonzero class in $H^{2}\left(X, v_{n}(1)\right)$ for all $n \geqslant 1$.

Proof. The first part is precisely [15, Corollary 5.7.1].

Proposition 9.2. Let $X$ be a classical Enriques surface over an algebraically closed field $k$, and let $\alpha \in \operatorname{Br}(X)$ denote the nonzero class. Then, $\operatorname{dlog} \alpha \neq 0$ in $H^{2}\left(X, \Omega_{X}^{1}\right)$.

Proof. Because $k$ is algebraically closed and $X$ is a smooth projective surface, we have an exact sequence

$$
0 \rightarrow \lim _{\longleftarrow} H^{2}\left(X, v_{\bullet}(1)\right) \stackrel{d \log ([-])}{\longrightarrow} H^{2}\left(X, W \Omega_{X}^{1}\right) \stackrel{1-F}{\longrightarrow} H^{2}\left(X, W \Omega_{X}^{1}\right) \rightarrow 0
$$

by $[22,5.22 .5]$.

Because $H^{2}\left(X, \mathcal{O}_{X}\right)=0$ and $H^{2}\left(X, W \mathcal{O}_{X}\right)=0$ (see Figures 2 and 3 reproduced from [22, Section II.7.3]), we have a commutative diagram

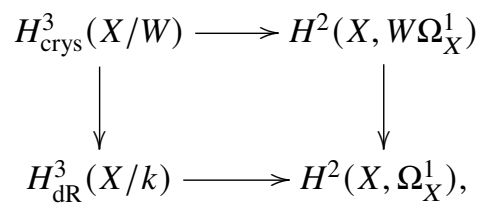

where the vertical maps are induced by killing $p$ in crystalline cohomology and the horizontal maps are the natural maps coming from the slope and Hodge filtrations. All four maps in the diagram are isomorphisms for $X$ a classical Enriques surface (again, see Figures 2 and 3). This shows that our class survives.

Thus, the twisted HKR spectral sequence does not degenerate for the nontrivial twist of a classical Enriques surface over an algebraically closed field of characteristic 2. More specifically, we can compute the Hochschild homology exactly.

Corollary 9.3. Let $\mathcal{A}$ be a geometrically nontrivial 2-torsion Azumaya algebra on a classical Enriques surface $X$ in characteristic 2 . Then, $H H(\mathcal{A} / k)$ is discrete and $H^{0}(H H(\mathcal{A} / k))$ is a 12-dimensional $k$ vector space. 


$\begin{array}{cccc} & \Omega_{X}^{0} & \Omega_{X}^{1} & \Omega_{X}^{2} \\ H^{2} & 0 & k & k \\ H^{1} & 0 & k^{12} & 0 \\ H^{0} & k & k & 0\end{array}$

Figure 2. The Hodge cohomology of a classical Enriques surface reproduced from [22, 7.3.8].

$\begin{array}{cccc} & W \Omega_{X}^{0} & W \Omega_{X}^{1} & W \Omega_{X}^{2} \\ H^{2} & 0 & k & W \\ H^{1} & 0 & W^{10} \oplus k & 0 \\ H^{0} & W & 0 & 0\end{array}$

Figure 3. The Hodge-Witt cohomology of a classical Enriques surface reproduced from [22, 7.3.6].

Proof. We can assume that $k$ is algebraically closed. We obtain that $\operatorname{HH}(\mathcal{A} / k)$ is self-dual; for example, via the Mukai pairing [37]. Here we can more explicitly see this because $\mathcal{A}$ is Morita equivalent to $\mathcal{A}^{o p}$ (because $2[\mathcal{A}]=0$ in $\operatorname{Br}(X)$ ). In particular, for any integer $i$, we have $H^{i}(\operatorname{HH}(\mathcal{A} / k)) \cong$ $H^{-i}(\mathrm{HH}(\mathcal{A} / k))$. The differential $d_{2}^{\alpha}: H^{0}\left(X, \Omega_{X}^{0}\right) \rightarrow H^{2}\left(X, \Omega_{X}^{1}\right)$ is an isomorphism by Proposition 7.7, Proposition 9.2 and dimension considerations (see Figure 2). We see from Figure 2 that $H^{1}(\mathrm{HH}(\mathcal{A} / k))=$ 0 . Thus, $H^{-1}(\mathrm{HH}(\mathcal{A} / k))=0$ and thus the differential $d_{2}^{\alpha}: H^{0}\left(X, \Omega^{1}\right) \rightarrow H^{2}\left(X, \Omega_{X}^{2}\right)$ must be an isomorphism.

\section{The conic bundle over a classical Enriques surface}

Let $X$ be a classical Enriques surface over an algebraically closed field $k$ of characteristic 2 . Let $\alpha \in \operatorname{Br}(X) \cong \mathbf{Z} / 2$ be the nonzero class. Because $X$ is a surface over an algebraically closed field, $\operatorname{ind}(\alpha)=\operatorname{per}(\alpha)$ by [16], so $\alpha$ is represented by a quaternion algebra $D$ over the generic point. Because $X$ is a regular 2-dimensional scheme, $D$ spreads out to a degree 2 Azumaya algebra $\mathcal{A}$ over $X$. Let $P \rightarrow X$ be the Severi-Brauer scheme associated to $\mathcal{A}$; it is a $\mathbf{P}^{1}$-bundle locally trivial in the étale topology.

Calculation 10.1. There is a semiorthogonal decomposition

$$
\operatorname{Perf}(P) \simeq\langle\operatorname{Perf}(X), \operatorname{Perf}(\mathcal{A})\rangle .
$$

Thus, we can compute the Hochschild homology of $P$ using additivity. (This is basically Quillen's argument from [33, Section 9], but see also [8].) By [7], the HKR spectral sequence degenerates for $X$, so (by Figure 2) $H^{i}(\mathrm{HH}(X / k))$ vanishes for $i \notin\{-1,0,1\}$; it is one-dimensional $i= \pm 1$ and 14dimensional for $i=0$. By Corollary 9.3, the $k$-vector spaces $H^{i}(\mathrm{HH}(P / k))$ vanish for $i \notin\{-1,0,1\}$. They are one-dimensional for $i= \pm 1$. And, for $i=0$, it is a 26-dimensional vector space.

Calculation 10.2. Let $\pi: P \rightarrow X$ be the structure morphism. Then, $\pi^{*} L_{X} \rightarrow L_{P} \rightarrow L_{\pi}$ is exact. We find an exact sequence

$$
0 \rightarrow \pi^{*} \Omega_{X}^{1} \rightarrow \Omega_{P}^{1} \rightarrow \omega_{P / X} \rightarrow 0
$$

of vector bundles. Hence, we have an equivalence $\pi^{*} \Omega_{X}^{2} \otimes_{\mathcal{O}_{P}} \omega_{P / X} \simeq \Omega_{P}^{3}$.

We might hope based on the failure of twisted HKR that HKR also fails for $P$. However, the next result proves that in fact the HKR spectral sequence does degenerate.

Theorem 10.3. For the conic bundle P constructed above, the HKR spectral sequence degenerates at $E_{2}$. 
Proof. We know by the calculation above the dimension of each $k$-vector space $H^{i}(\mathrm{HH}(P / k))$. It is enough to check that

$$
\sum_{s+t=i} \operatorname{dim}_{k} H^{s}\left(P, \Omega_{P}^{-t}\right)=\operatorname{dim} H^{i}(\mathrm{HH}(P / k)) .
$$

We can do this calculation up to a small discrepancy for any $\mathbf{P}^{1}$-bundle over $X$. Resolving this discrepancy involves using the fact that the pullback map $\operatorname{Br}(X) \rightarrow \operatorname{Br}(P)$ kills $\alpha$.

We will fill out the table in Figure 4 for this threefold. By Serre duality, saying that $H^{s}\left(P, \Omega_{P}^{t}\right) \cong$ $H^{3-s}\left(P, \Omega_{P}^{3-t}\right)^{*}$, it is enough to fill out the first two columns. In the figure, $\epsilon$ refers to a fixed number, either 0 or 1 ; it is the same number each place it appears.

Because $\pi: P \rightarrow X$ is a $\mathbf{P}^{1}$-bundle, we find that $R \pi_{*} \mathcal{O}_{P} \simeq \mathcal{O}_{X}$. Hence, $H^{s}\left(P, \pi^{*} \Omega_{X}^{t}\right) \cong H^{s}\left(X, \Omega_{X}^{t}\right)$ by adjunction for all $s, t$. Moreover, we see immediately that $H^{s}\left(P, \mathcal{O}_{P}\right)=0$ for $s>0$. This computes the first column. For the second, we use the conormal sequence. Let us first compute the cohomology of $\omega_{P / X}$. By Grothendieck-Verdier duality for $P \rightarrow X$, we see that $R \pi_{*} \omega_{P / X} \simeq \mathcal{O}_{X}[-1]$. Therefore, using the spectral sequence

$$
E_{2}^{a, b}=H^{a}\left(X, R^{b} \pi_{*} \omega_{P / X}\right) \Rightarrow H^{a+b}\left(P, \omega_{P / X}\right),
$$

we see that $H^{s}\left(P, \omega_{P / X}\right) \cong H^{s-1}\left(X, \mathcal{O}_{X}\right)$. In other words, using Figure 2, we have that $H^{s}\left(P, \omega_{P / X}\right)$ is one-dimensional for $s=1$ and zero otherwise. The long exact sequence for the cohomology of $0 \rightarrow \pi^{*} \Omega_{X}^{1} \rightarrow \Omega_{P}^{1} \rightarrow \omega_{P / X} \rightarrow 0$ gives us an exact sequence

$$
0 \rightarrow H^{1}\left(X, \Omega_{X}^{1}\right) \rightarrow H^{1}\left(P, \Omega_{P}^{1}\right) \rightarrow k \rightarrow H^{2}\left(X, \Omega_{X}^{1}\right) \rightarrow H^{2}\left(P, \Omega_{P}^{1}\right) \rightarrow 0
$$

as well as isomorphisms

$$
\begin{aligned}
& H^{0}\left(X, \Omega_{X}^{1}\right) \cong H^{0}\left(P, \Omega_{P}^{1}\right) \\
& H^{3}\left(X, \Omega_{X}^{1}\right) \cong H^{3}\left(P, \Omega_{P}^{1}\right) .
\end{aligned}
$$

Hence, $H^{0}\left(P, \Omega_{P}^{1}\right) \cong k$ and $H^{3}\left(P, \Omega_{P}^{1}\right)=0$.

Using the exact sequence, we see that $\epsilon=1$ if $H^{1}\left(P, \Omega_{P}^{1}\right) \rightarrow k$ is surjective (bearing in mind that $H^{2}\left(X, \Omega_{X}^{1}\right)$ is one-dimensional). We have $\epsilon=0$ if $H^{2}\left(X, \Omega_{X}^{1}\right) \rightarrow H^{2}\left(P, \Omega_{P}^{1}\right)$ is zero.

This completes the analysis of the table. To determine $\epsilon$, we use the commutative diagram

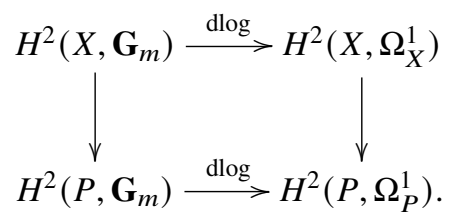

Because the pullback map $H^{2}\left(X, \mathbf{G}_{m}\right) \rightarrow H^{2}\left(P, \mathbf{G}_{m}\right)$ kills $\alpha$, by definition of the Severi-Brauer scheme, and because dlog $\alpha$ is nonzero in $H^{2}\left(X, \Omega_{X}^{1}\right)$, we see that $H^{2}\left(X, \Omega_{X}^{1}\right) \rightarrow H^{2}\left(P, \Omega_{P}^{1}\right)$ has a nontrivial

$\begin{array}{ccccc} & \Omega_{P}^{0} & \Omega_{P}^{1} & \Omega_{P}^{2} & \Omega_{P}^{3} \\ H^{3} & 0 & 0 & k & k \\ H^{2} & 0 & k^{0+\epsilon} & k^{12+\epsilon} & 0 \\ H^{1} & 0 & k^{12+\epsilon} & k^{0+\epsilon} & 0 \\ H^{0} & k & k & 0 & 0\end{array}$

Figure 4. The Hodge cohomology of the conic bundle over a classical Enriques surface. 
kernel and hence is identically zero because the map is $k$-linear and $H^{2}\left(X, \Omega_{X}^{1}\right)$ is one-dimensional over $k$. Thus, we get $\epsilon=0$ and a dimension count now completes the proof.

We see from this a phenomenon that only exists in characteristic $p>0$ : Hodge cohomology can distinguish between $\mathbf{P}^{n}$-bundles. To begin with, we observe that this never happens in characteristic 0 .

Proposition 10.4 (Hodge cohomology of $\mathbf{P}^{n}$-bundles). Suppose that $k$ is a field of characteristic 0 and $X$ is a smooth $k$-scheme. Let $\pi: P \rightarrow X$ be a Severi-Brauer scheme (i.e., an étale locally trivial $\boldsymbol{P}^{n}$-bundle). The Hodge cohomology $H^{*}\left(P, \Omega_{P}^{*}\right)$ is a free bigraded module over $H^{*}\left(X, \Omega_{X}^{*}\right)$ on the set $\left\{1, c, \ldots, c^{n}\right\}$, where $c=c_{1}\left(\omega_{P / X}\right) \in H^{1}\left(P, \Omega_{P}^{1}\right)$.

This proposition may be regarded as an instance of the Leray-Hirsch theorem for Hodge cohomology. As the following proof shows, the result also holds true in characteristic $p$ provided $p \nmid(n+1)$.

Proof. As in the proof of Proposition 5.11, because the formation of $\omega_{P / X}$ and $c$ commutes with base change on $X$, we may work étale locally on $X$ to reduce to the case $P=\mathbf{P}^{n} \times X$. By the Künneth formula, $H^{*}\left(P, \Omega_{P}^{*}\right)$ is a free bigraded module over $H^{*}\left(X, \Omega_{X}^{*}\right)$ on the set $\left\{1, d, \ldots, d^{n}\right\}$, where $d=c_{1}\left(\operatorname{pr}_{1}^{*} \mathcal{O}_{\mathbf{P}^{n}}(1)\right)$. Because $\omega_{P / X} \simeq \operatorname{pr}_{1}^{*} \mathcal{O}_{\mathbf{P}^{n}}(-n-1)$, we have $c=-(n+1) d$. Because we are working in characteristic 0 , it is then clear that $H^{*}\left(P, \Omega_{P}^{*}\right)$ is then also a free bigraded module over $H^{*}\left(X, \Omega_{X}^{*}\right)$ on the set $\left\{1, c, \ldots, c^{n}\right\}$.

For $\mathbf{P}^{n}$-bundles that arise as projectivisations of vector bundles, Proposition 10.4 holds in arbitrary characteristic as in Proposition 5.11. By contrast, in characteristic $p>0$, we see that Hodge cohomology can distinguish between $\mathbf{P}^{n}$-bundles $P$ and $\mathbf{P}_{X}^{n}$ in some cases. If $X$ is a smooth proper variety over any field $k$ and $\pi: P \rightarrow X$ is a $\mathbf{P}^{n}$-bundle, then a slight elaboration of the above proof (to handle $R \pi_{*} \Omega_{P}^{i}$ ) shows that the Hodge cohomology of $P$ is bounded above by that of $\mathbf{P}_{X}^{n}$. However, this bound may be strict. For example, in characteristic 2, we have seen that the pullback in Hodge cohomology the conic bundle for $P \rightarrow X$ over a classical Enriques surface $X$ is not injective; specifically, $H^{2}\left(X, \Omega_{X}^{1}\right) \rightarrow H^{2}\left(P, \Omega_{P}^{1}\right)$ was not injective thanks to the dlog map. In characteristic $p>2$, the results of Section 8 show that there is a smooth projective threefold $Y$ and a $\mathbf{P}^{p-1}$-bundle $\pi^{\prime}: P^{\prime} \rightarrow Y$ such that the pullback $H^{2}\left(Y, \Omega_{Y}^{1}\right) \rightarrow H^{2}\left(P^{\prime}, \Omega_{P^{\prime}}^{1}\right)$ is not injective.

Acknowledgements. We thank Daniel Bragg, Lukas Brantner, Johan de Jong, Hélène Esnault, Marci Hablicsek, Daniel HalpernLeistner, Yankı Lekili, Alexander Petrov, Bertrand Toën, Burt Totaro and Gabriele Vezzosi for discussions about HKR in characteristic $p$. This material is based partially on work supported by the National Science Foundation under Grant No. DMS1440140 while the authors were in residence at the Mathematical Sciences Research Institute in Berkeley, California, during the Spring 2019 semester. The first author was partially supported by NSF Grant DMS-1552766. The second author was partially supported by the NSF grants \#1501461 and \#1801689, a Packard fellowship and the Simons Foundation grant \#622511. This work was done while the third author was a Clay Research Fellow.

Conflict of Interest: None.

\section{References}

[1] B. Antieau, 'Čech approximation to the Brown-Gersten spectral sequence', Homology Homotopy Appl. 13(1) (2011), 319_ 348 .

[2] B. Antieau, 'Cohomological obstruction theory for Brauer classes and the period-index problem', J. K-Theory 8(3) (2011), 419-435.

[3] B. Antieau, 'Periodic cyclic homology and derived de Rham cohomology', Ann. K-Theory 4(3) (2019), 505-519.

[4] B. Antieau and D. Bragg, 'Derived invariants from topological Hochschild homology', Preprint, 2019, arXiv:1906.12267.

[5] B. Antieau and D. Gepner, 'Brauer groups and étale cohomology in derived algebraic geometry', Geom. Topol. 18(2) (2014), 1149-1244.

[6] B. Antieau, A. Mathew and T. Nikolaus, 'On the Blumberg-Mandell Künneth theorem for TP', Selecta Math. (N.S.) 24(5) (2018), 4555-4576.

[7] B. Antieau and G. Vezzosi, 'A remark on the Hochschild-Kostant-Rosenberg theorem in characteristic p', Ann. Sc. Norm. Super. Pisa Cl. Sci. (5) 20(3) (2020), 1135-1145.

[8] M. Bernardara, 'A semiorthogonal decomposition for Brauer-Severi schemes', Math. Nachr. 282(10) (2009), $1406-1413$. 
[9] B. Bhatt, 'Completions and derived de Rham cohomology', Preprint, 2012, arXiv:1207.6193.

[10] B. Bhatt, ' $p$-Adic derived de Rham cohomology', Preprint, 2012, arXiv:1204.6560.

[11] B. Bhatt, M. Morrow and P. Scholze, 'Integral p-adic Hodge theory', Publ. Math. Inst. Hautes Études Sci. 128 (2018), 219-397.

[12] B. Bhatt, M. Morrow and P. Scholze, 'Topological Hochschild homology and integral p-adic Hodge theory', Publ. Math. Inst. Hautes Études Sci. 129 (2019), 199-310.

[13] E. Bombieri and D. Mumford, 'Enriques' classification of surfaces in char. p. III', Invent. Math. 35 (1976), $197-232$.

[14] G. Cortiñas and C. Weibel, 'Homology of Azumaya algebras', Proc. Amer. Math. Soc. 121(1) (1994), 53-55.

[15] F. R. Cossec and I. V. Dolgachev, Enriques Surfaces. I, Progress in Mathematics, Vol. 76 (Birkhäuser, Boston, 1989).

[16] A. J. de Jong, 'The period-index problem for the Brauer group of an algebraic surface', Duke Math. J. 123(1) (2004), 71-94.

[17] P. Deligne and L. Illusie, 'Relèvements modulo $p^{2}$ et décomposition du complexe de de Rham', Invent. Math. 89(2) (1987), 247-270.

[18] P. Deligne and N. Katz. II, Lecture Notes in Mathematics, Vol. 340 (Springer, Berlin, 1973).

[19] E. M. Friedlander, 'Lectures on the cohomology of finite group schemes', in Rational Representations, the Steenrod Algebra and Functor Homology, Panor. Synthèses, Vol. 16 ( Société Mathématique de France, Paris, 2003), pp. $27-53$.

[20] M. Gerstenhaber and S. D. Schack, 'A Hodge-type decomposition for commutative algebra cohomology', J. Pure Appl. Algebra 48(3) (1987), 229-247.

[21] L. Illusie, Complexe cotangent et déformations. II, Lecture Notes in Mathematics, Vol. 283 (Springer, Berlin, 1972).

[22] L. Illusie, 'Complexe de de Rham-Witt et cohomologie cristalline', Ann. Sci. École Norm. Sup. (4) 12(4) (1979), 501-661.

[23] L. Illusie, 'Frobenius et dégénérescence de Hodge', in Introduction à la théorie de Hodge, Panor. Synthèses, Vol. 3 (Société Mathématique de France, Paris), 1996, pp. 113-168.

[24] J. C. Jantzen, Representations of Algebraic Groups, Pure and Applied Mathematics, Vol. 131 (Academic Press, Boston, 1987).

[25] D. Kaledin, 'Non-commutative Hodge-to-de Rham degeneration via the method of Deligne-Illusie', Pure Appl Math Q. 4(3) (2008), 785-876.

[26] D. Kaledin, 'Spectral sequences for cyclic homology', in Algebra, Geometry, and Physics in the 21st Century (Springer, Basel, 2017), pp. 99-129.

[27] J. Lurie, Higher Topos Theory, Annals of Mathematics Studies, Vol. 170 (Princeton University Press, Princeton, NJ, 2009).

[28] J. Lurie, 'Spectral algebraic geometry', (2018). URL: http://www.math.harvard.edu/ lurie/.

[29] A. Mathew, 'Kaledin's degeneration theorem and topological Hochschild homology', Geom. Topol. 24(6) (2020), 26752708.

[30] T. Nikolaus and P. Scholze, 'On topological cyclic homology', Acta Math. 221(2) (2018), 203-409.

[31] B. Poonen, 'Bertini theorems over finite fields', Ann. Math. (2) 160(3) (2004), 1099-1127.

[32] D. Quillen, 'On the (co-) homology of commutative rings', in Applications of Categorical Algebra (Proc. Sympos. Pure Math., Vol. XVII, New York, 1968) (American Mathematical Society, Providence, RI, 1970), pp. 65-87.

[33] D. Quillen, 'Higher algebraic K-theory. I', in Algebraic K-theory, I: Higher K-theories (Proc. Conf., Battelle Memorial Inst., Seattle, Wash., 1972), Lecture Notes in Mathematics (Berlin, Heidelberg), Vol. 341 (1973), pp. 85-147.

[34] S. Rao, S. Yang, X. Yang and X. Yu, 'Hodge cohomology on blow-ups along subvarieties', Preprint, 2019, arXiv:1907.13281.

[35] J. Rosenberg, 'Algebraic K-theory and its applications', in Graduate Texts in Mathematics, Vol. 147 (Springer, New York, 1994).

[36] J.-P. Serre, 'Sur la topologie des variétés algébriques en caractéristique p', in Symposium internacional de topología algebraica [International symposium on algebraic topology] (Universidad Nacional Autónoma de México and UNESCO, Mexico City, 1958), pp. 24-53.

[37] D. Shklyarov, 'Hirzebruch-Riemann-Roch-type formula for DG algebras', Proc. Lond. Math. Soc. (3) 106(1) (2013), 1-32.

[38] The Stacks Project Authors, 'The Stacks Project', (2019). URL: https://stacks.math.columbia.edu.

[39] B. Toën, 'Derived Azumaya algebras and generators for twisted derived categories', Invent. Math. 189(3) (2012), 581-652.

[40] B. Toën and G. Vezzosi, 'Algèbres simpliciales $S^{1}$-équivariantes, théorie de de Rham et théorèmes HKR multiplicatifs', Compos. Math. 147(6) (2011), 1979-2000.

[41] B. Totaro, 'The Chow ring of a classifying space', in Algebraic K-Theory, Proc. Sympos. Pure Math., Vol. 67 (American Mathematical Society, Providence, RI, 1999), pp. 249-281.

[42] B. Totaro, 'Hodge theory of classifying stacks', Duke Math. J. 167(8) (2018), 1573-1621.

[43] C. Weibel, 'The Hodge filtration and cyclic homology', K-Theory 12(2) (1997), 145-164.

[44] C. A. Weibel and S. C. Geller, 'Étale descent for Hochschild and cyclic homology', Comment. Math. Helv. 66(3) (1991), 368-388.

[45] A. Yekutieli, 'The continuous Hochschild cochain complex of a scheme', Can. J. Math. 54(6) (2002), $1319-1337$. 